\title{
MODERATE DEVIATION FOR RANDOM ELLIPTIC PDE WITH SMALL NOISE
}

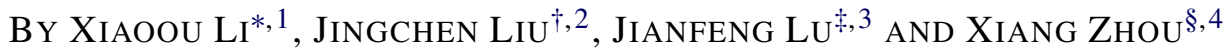 \\ University of Minnesota*, Columbia University ${ }^{\dagger}$, Duke University ${ }^{\ddagger}$ and \\ City University of Hong Kong $\$$
}

\begin{abstract}
Partial differential equations with random inputs have become popular models to characterize physical systems with uncertainty coming from imprecise measurement and intrinsic randomness. In this paper, we perform asymptotic rare-event analysis for such elliptic PDEs with random inputs. In particular, we consider the asymptotic regime that the noise level converges to zero suggesting that the system uncertainty is low, but does exist. We develop sharp approximations of the probability of a large class of rare events.
\end{abstract}

1. Introduction. The study of rare events due to system uncertainty, for example, the failure of materials due to intrinsic randomness, is crucial and yet challenging. While those events do not often occur, they lead to catastrophic consequences. Therefore, it is important to estimate the probabilities of such events and to characterize those events which help finding interventions to prevent them from happening. In this paper, we consider the following classical continuum mechanical model in the form of a linear elliptic partial differential equation (PDE) defined on a domain $U \subset \mathbb{R}^{d}$,

$$
-\nabla \cdot(a(x) \nabla u(x))=f(x),
$$

subject to certain boundary conditions that will be specified in the sequel. The solution $u$ to the above equation describes the deformation of a piece of elastic material under the external force $f$. The function $a(x)$ is known as the elasticity determined by the property of the specific material and it is strictly positive. Instead of assuming that $a$ is deterministic, we are interested in the situations when the tensor $a$ contains randomness. The randomness is introduced to incorporate the uncertainties of simple elastic materials at the macroscopic level or heterogeneity in the microstructures of complex materials. Under this setting, the solution $u(x)$

Received October 2016; revised November 2017.

${ }^{1}$ Supported in part by the National Science Foundation (DMS-1712657).

${ }^{2}$ Supported in part by the National Science Foundation (SES-1323977, IIS-1633360) and Army Grant (W911NF-15-1-0159).

${ }^{3}$ Supported in part by National Science Foundation (DMS-1454939).

${ }^{4}$ Supported by Hong Kong General Research Fund (109113, 11304314, 11304715).

MSC2010 subject classifications. Primary 60F10, 60Z05; secondary 60G15.

Key words and phrases. Random partial differential equation, rare event, moderate deviation. 
[as a function of $a(x)$ ] is also a stochastic process whose law is determined by that of $a(x)$.

Besides material mechanics, the elliptic PDE (1) arises also in many other fields of applications, such as hydrogeology and porous medium. The tensor $a(x)$ carries different names such as conductivity and permeability. It is recognized that the modeling of the random field $a(x)$ is of primal importance for the analysis. In this paper, we consider the following stylized model:

$$
a(x)=a_{0}(x) e^{-\sigma \xi(x)}, \quad x \in U,
$$

where $\xi(x)$ is a Gaussian random field defined on $U$ and $a_{0}(x)$ is a deterministic function. The scalar $\sigma>0$ is a parameter indexing the noise level. Many studies by practitioners, for example, Freeze [(1975), pages 728-729] and Charbeneau [(2000), page 40], have shown that the log-normal distribution provides a good fit to the empirical data. Hence, the log-normal assumption is well justified in applications and is used in mathematical analysis and numerical computation of the random PDE (1). In our paper, we follow this convention of log-normal assumption for the rare-event analysis.

In this work, we consider the small noise asymptotic regime that $\sigma$ tends to zero. Yet, even small noise can lead to a drastic difference of the PDE solution from that of the deterministic case when the noise level is zero. Our results characterize such rare events, more precisely, the deviation of the solution of the random elliptic PDE in presence of small noise. In particular, we focus on the deviation from the deterministic solution as the uncertainty level goes to 0 . Let $\mathcal{H}$ be a mapping from $C(\bar{U})$ to $\mathbb{R}$. Of primary interest is

$$
\omega(\sigma)=\mathbb{P}\left\{\mathcal{H}(u)>\mathcal{H}\left(u_{0}\right)+b_{\sigma}\right\} \quad \text { as } \sigma \rightarrow 0,
$$

where $u$ is the solution to equation (1) and $u_{0}$ is the solution when the noise level is zero, that is, $a(x)=a_{0}(x)$. The level $b_{\sigma}$ will be sent to zero as the noise level $\sigma$ goes to zero, which will be specified in the sequel. The main contribution of this paper is to derive sharp closed-form asymptotic approximations of $\omega(\sigma)$ as $\sigma \rightarrow 0$. To the authors best knowledge, this is the first rigorous asymptotic analysis of random elliptic PDE for $d>1$.

Given that $\mathcal{H}(u)$ is a (complicated) functional of the input Gaussian process $\xi(x)$, the analysis of the tail probability $\omega(\sigma)$ links naturally to the rare-event analysis of Gaussian random field. The study of the extremes of Gaussian random fields focuses mostly on the tail probabilities of the supremum of the field. The results contain general bounds on $P(\max \xi(x)>b)$ as well as sharp asymptotic approximations as $b \rightarrow \infty$. A partial literature contains Landau and Shepp (1970), Marcus and Shepp (1970), Sudakov and Tsirelson (1974), Borell (1975, 2003), Berman (1985), Ledoux and Talagrand (1991), Talagrand (1996). Several methods have been introduced to obtain bounds and asymptotic approximations. A gen- 
eral upper bound for the tail of $\max \xi(x)$ is developed in Borell (1975), Cirel'son, Ibragimov and Sudakov (1976), which is known as the Borel-TIS inequality. For asymptotic results, there are several methods, such as the double sum method [Piterbarg (1996)], the Euler-Poincaré characteristics of the excursion set approximation [Adler (1981), Adler and Taylor (2007), Taylor and Adler (2003), Taylor, Takemura and Adler (2005)], the tube method [Sun (1993)] and the Rice method [Azaiis and Wschebor (2008, 2009)]. Recently, the exact tail approximation of integrals of exponential functions of Gaussian random fields is developed by Liu (2012), Liu and Xu (2012). Efficient computations via importance sampling have been developed by Adler, Blanchet and Liu (2008, 2012). For the analysis of the tail probabilities of log-normal random fields with small noise, refer to the recent work in Li, Liu and $\mathrm{Xu}$ (2016).

There are also existing work in the context of PDE with random coefficients. Liu and Zhou $(2013,2014)$ study the asymptotic behavior of one-dimensional $(d=1)$ elliptic PDE. Liu, Lu and Zhou (2015) presents the corresponding rareevent simulation algorithms. The main difference between the cases $d=1$ and $d>1$ is that $u(x)$ can be written analytically as a function of $a(x)$ for $d=1$ and there is no closed-form solution of $u(x)$ for $d>1$. Furthermore, $\mathrm{Xu}$, Lin and Liu (2014) presents asymptotic analysis for the stochastic KdV equation. Recently, Berglund et al. (2017) presents analysis on an Eyring-Kramers law for an Allen-Cahn equation driven by weak space-time white noise when $d=2$. The recent book Armstrong, Kuusi and Mourrat (2017) discusses stochastic homogenization of elliptic operators with random coefficients. In particular, Theorem 2.15 of Armstrong, Kuusi and Mourrat (2017) could be interpreted as a large deviation estimate, and Theorem 5.24 of Armstrong, Kuusi and Mourrat (2017) presents a central limit theory type of result.

The rest of the paper is organized as follows. Section 2 presents the problem setup and the main asymptotic results. The technical proofs are given in Section 3.

\section{Main results.}

2.1. The problem setup. We consider the following elliptic PDE. Let $U \subset \mathbb{R}^{d}$ be an open, bounded domain with a smooth boundary. The differential equation concerning $u: U \rightarrow \mathbb{R}$ with Dirichlet boundary condition is given by

$$
\begin{cases}-\nabla \cdot(a(x) \nabla u(x))=f(x) & \text { for } x \in U ; \\ u(x)=0 & \text { for } x \in \partial U .\end{cases}
$$

In the context of elastic mechanics, $u$ characterizes the material deformation due to external force $f$ and $a: U \rightarrow \mathbb{R}$ gives the stiffness of the material. We assume that the material is clamped to a frame on the boundary $\partial U$, and hence the Dirichlet boundary condition $u(\partial U)=0$ in (3) is assumed. The external force $f$ is sufficiently smooth and bounded, that is, there exists a constant $c \in \mathbb{R}$ such that

$$
|f(x)| \leq c \quad \forall x \in U
$$


We study the behavior of the material under the influence of internal randomness, which may be the result of manufacturing processing or the uncertainty of the material properties at the microscopic level. We adopt a probabilistic viewpoint of the complexity and heterogeneity inherent in the material and view the coefficient $a(x)$ as a random field. The process $a(x)$ is physically restricted to be positive and is modeled as a log-normal random field given as in (2). Furthermore, the Gaussian random function $\xi$ has mean zero and its covariance function is denoted by

$$
C(x, y)=\mathbf{E}\{\xi(x) \xi(y)\},
$$

which does not depend on $\sigma$.

The solution $u(x)$ depends implicitly on $a(x)$ through equation (3) and further $\xi(x)$ via a logarithmic change of variable. It is useful to define a mapping from the coefficient $\xi$ to the solution $u$

$$
\mathbf{J}[\xi] \triangleq u_{\xi},
$$

where $u_{\xi}$ is the solution to equation (3) with $a(x)=a_{0}(x) e^{-\xi(x)}$. This mapping depends only on the deterministic function $a_{0}$, the external force $f$, the domain $U$ and the boundary condition. In this paper, we are interested in the asymptotic regime that the amplitude of the uncertainty level $\sigma$ tends to zero. Then the failure problem concerns the random solution $u_{\sigma \xi}=\mathbf{J}(\sigma \xi)$ by noting the definition of $\mathbf{J}$ above. As $\sigma \rightarrow 0$, the process $a(x)$ tends to its limiting field $a_{0}(x)$. Let $u_{0}(x)$ be the corresponding limiting solution satisfying equation

$$
\begin{cases}-\nabla \cdot\left(a_{0}(x) \nabla u_{0}(x)\right)=f(x) & \text { for } x \in U ; \\ u_{0}(x)=0 & \text { for } x \in \partial U .\end{cases}
$$

Then, under mild conditions, we have $u(x) \rightarrow u_{0}(x)$ as $\sigma \rightarrow 0$.

We provide asymptotic analysis of the event that $u$ deviates from its limiting solution $u_{0}$. Let $\mathcal{H}$ be a functional from $C(\bar{U})$ to $\mathbb{R}$ characterizing the deviation. For instance, $\mathcal{H}(u)=\int_{U}\left(u(x)-u_{0}(x)\right) d x$. Let $\mathcal{G}$ be the composition of $\mathbf{J}$ and $\mathcal{H}$, that is,

$$
\mathcal{G}(\xi)=\mathcal{H}(\mathbf{J}[\xi]) \text {. }
$$

To simplify notation, we always choose $\mathcal{H}$ such that $\mathcal{G}(\mathbf{0})=\mathcal{H}\left(u_{0}\right)=0$. We are interested the tail probability of $\mathcal{G}(\sigma \xi)$ as $\sigma \rightarrow 0$. In particular, we derive asymptotic approximations for

$$
\omega(\sigma)=\mathbb{P}\{\mathcal{G}(\sigma \xi)>b\} \quad \text { as } \sigma \rightarrow 0,
$$

where the deviation level is chosen to be $b=\kappa \sigma^{\alpha}$ for some fixed $\alpha \in(0,1)$ and $\kappa>0$. In particular, the deviation level $b$ also goes to 0 as the uncertainty vanishes. Without loss of generality, we fix $\kappa=1$ and $b=\sigma^{\alpha}$ in the following discussion, as $\kappa$ can be absorbed in the functional $\mathcal{G}$. 
2.2. Notation. We first introduce some notation that will be used in the sequel. Throughout this analysis, we consider $\mathcal{G}$ to be a differentiable functional and let $\mathcal{G}^{\prime}$ be its Fréchet derivative, that is,

$$
\mathcal{G}(\xi+\varepsilon \eta)=\mathcal{G}(\xi)+\varepsilon \int_{U} \mathcal{G}^{\prime}[\xi](x) \eta(x) d x+o(\varepsilon) \quad \text { as } \varepsilon \rightarrow 0, \forall \xi, \eta \in C(\bar{U}) .
$$

For $0<\beta<1$, we say that a function $w$ is Hölder continuous with order $\beta$ if the Hölder coefficient

$$
[w]_{\beta}=\sup _{x, y \in \bar{U}, x \neq y} \frac{|w(x)-w(y)|}{|x-y|^{\beta}}<\infty .
$$

We use $C^{k}(\bar{U})$ to denote the space containing all $k$-time continuously differentiable functions. For nonnegative integer $k$ and $0 \leq \beta<1$, we use $C^{k, \beta}(\bar{U})$ to denote the set of functions in $C^{k}(\bar{U})$ whose $k$ th order partial derivatives are Hölder continuous with coefficient $\beta$. For simplicity, we write $C^{0, \beta}(\bar{U})=C^{\beta}(\bar{U})$. We proceed to the definition of norms over $C^{k, \beta}(\bar{U})$. We first define the seminorms

$$
[w]_{k, 0}=\max _{|\gamma|=k} \sup _{\bar{U}}\left|D^{\gamma} w\right| \quad \text { and } \quad[w]_{k, \beta}=\max _{|\gamma|=k}\left[D^{\gamma} w\right]_{\beta}
$$

where $\gamma$ is a multi-index $\gamma=\left(\gamma_{1}, \ldots, \gamma_{d}\right),|\gamma|=\sum_{i=1}^{d} \gamma_{i}$, and $D^{\gamma} w=$ $\frac{\partial^{|\gamma|} w}{\partial^{\gamma 1} x_{1} \cdots \partial^{\gamma d} x_{d}}$. We further define the norms

$$
\|w\|_{C^{k}(\bar{U})}=\sum_{j=0}^{k}[w]_{j, 0} \quad \text { and } \quad\|w\|_{C^{k, \beta}(\bar{U})}=\|w\|_{C^{k}(\bar{U})}+[w]_{k, \beta} .
$$

Equipped with $\|\cdot\|_{C^{k, \beta}(\bar{U})}$, the space $C^{k, \beta}(\bar{U})$ is a Banach space for all nonnegative integer $k$ and $0 \leq \beta<1$. To simplify notation, we write

$$
|w|_{k}=\|w\|_{C^{k}(\bar{U})}, \quad|w|_{k, \beta}=\|w\|_{C^{k, \beta}(\bar{U})}, \quad|w|_{\beta}=|w|_{0, \beta} .
$$

2.3. Asymptotic results. We make the following assumptions on the smoothness of functional $\mathcal{H}$ and the elliptic PDE, as well as the covariance function $C(x, y)$.

H1. There exist constants $\beta, \delta_{H}, \kappa_{H}$ such that $\delta_{H}>0,0<\beta<1$ and $\mathcal{H}^{\prime}(u) \in$ $C^{\beta}(\bar{U})$ for all $\left|u-u_{0}\right|_{2, \beta} \leq \delta_{G}$. In addition, $\mathcal{H}^{\prime}$ is Lipschitz in the sense that

$$
\left|\mathcal{H}^{\prime}\left[u_{1}\right]-\mathcal{H}^{\prime}\left[u_{2}\right]\right|_{\beta} \leq \kappa_{H}\left|u_{1}-u_{2}\right|_{2, \beta}
$$

for all $\left|u_{1}-u_{0}\right|_{2, \beta},\left|u_{2}-u_{0}\right|_{2, \beta} \leq \delta_{H}$. Here, $u_{0} \in C^{2, \beta}(\bar{U})$ is the solution to (6) when $\xi$ is set to be $\mathbf{0}$.

H2. There exists $x \in \bar{U}$ such that $\nabla g_{0}(x) \cdot \nabla u_{0}(x) \neq 0$, where $g_{0} \in C^{2, \beta}(\bar{U})$ is the solution to the PDE

$$
\begin{cases}-\nabla \cdot\left(a_{0}(x) \nabla g_{0}(x)\right)=\mathcal{H}^{\prime}\left[u_{0}\right](x) & \text { for } x \in U \\ g_{0}(x)=0 & \text { for } x \in \partial U\end{cases}
$$


H3. The set $U$ is a bounded domain with a $C^{2, \beta}$ boundary $\partial U, a_{0} \in C^{1, \beta}(\bar{U})$, $\min _{x \in \bar{U}} a_{0}(x)>0$ and $f \in C^{\beta}(\bar{U})$.

H4. The Gaussian random field $\{\xi(x), x \in U\}$ belongs to the space $C^{1, \beta}(\bar{U})$ almost surely. Its covariance function $C(\cdot, \cdot)$ is positive definite and satisfies $\sup _{y \in \bar{U}}|C(\cdot, y)|_{1,2 \beta}<\infty$. Moreover, we assume that for all $\gamma$ such that $|\gamma| \leq 1$, $\sup _{y \in \bar{U}}\left|C_{D^{\gamma} \xi}(\cdot, y)\right|_{2 \beta}<\infty$, where $C_{D^{\gamma} \xi}$ is defined in (14).

Define a mapping $\mathbf{C}: C(\bar{U}) \rightarrow C(\bar{U})$

$$
\mathbf{C} w \triangleq \int C(\cdot, y) w(y) \mathrm{d} y .
$$

We consider the optimization problem

$$
K_{\sigma}^{*}=\min _{\xi \in \mathcal{B}, \mathcal{G}(\sigma \mathbf{C} \xi)=\sigma^{\alpha}} \mathcal{K}(\xi),
$$

where the functional $\mathcal{K}: C(\bar{U}) \rightarrow \mathbb{R}$ is

$$
\mathcal{K}(w) \triangleq \int_{U} w(x) C(x, y) w(y) d x d y,
$$

and the set $\mathcal{B}$ is defined as

$$
\mathcal{B} \triangleq\left\{w \in C^{k, \beta}(\bar{U}):|w|_{k, \beta} \leq \sigma^{\alpha-1-\varepsilon}\right\}
$$

for some $k \geq 0, \varepsilon \in(0, \min (\alpha / 2,(1-\alpha) / 2))$ and $\alpha$ is given as below (8). Because $\mathcal{B}$ is a compact subset of $C^{k, \beta}(\bar{U})$ and the functionals $\mathcal{K}$ and $\mathcal{G}$ are continuous over $\mathcal{B}$, the above optimization problem has at least one solution. Later in the current section, we will show that this solution is also unique. The sharp asymptotic approximation for the elliptic PDE with small noise is presented in the next theorem.

THEOREM 1. Under the Assumptions $\mathrm{H} 1-\mathrm{H} 4$, for $0<\alpha<1$, we have

$$
\mathbb{P}\left\{\mathcal{G}(\sigma \xi)>\sigma^{\alpha}\right\}=\left(c_{1}+o(1)\right) \sigma^{1-\alpha} \exp \left(-\frac{1}{2} K_{\sigma}^{*}\right) \quad \text { as } \sigma \rightarrow 0,
$$

where $c_{1}=\left\{(2 \pi)^{-1} \mathcal{K}\left(a \nabla g_{0} \cdot \nabla u_{0}\right)\right\}^{\frac{1}{2}}$ and $K_{\sigma}^{*}$ is the minimum obtained in (11) with $k=1$ in (13) and $\beta$ being defined as in Assumptions $\mathrm{H} 1-\mathrm{H} 4$.

Under Assumption H3, the PDE (3) has a unique solution $u_{0} \in C^{2, \beta}(\bar{U})$ when $\xi$ is set to be 0. Furthermore, under Assumptions H1 and H3, (10) also has a unique solution in $C^{2, \beta}(\bar{U})$. Therefore, $g_{0}$ and $u_{0}$ in the above theorem are well-defined; see Lemma 6 on page 2798 for the existence and the uniqueness of the Hölder continuous solution to elliptic PDEs.

The solution of the optimization in (11) is generally not in a closed form, and so is $K_{\sigma}^{*}$. In what follows, we present results on asymptotic approximation of $K_{\sigma}^{*}$ as well as its numerical approximation. Our strategy for developing these results and proving Theorem 1 is to consider a general functional $\mathcal{G}$, which is not necessary to be $\mathcal{H}(\mathbf{J})$ as defined in (7), and develop general theories accordingly. 
2.4. Asymptotic results for general functionals. We present sharp asymptotic approximations of the tail probabilities $w(\sigma)$ under the following assumptions on a functional $\mathcal{G}$, which is not necessary in the form as is defined in (7), and the covariance function $C(x, y)$.

\section{ASSUMPTION.}

A1. There exist constants $k, \beta, \delta_{G}, \kappa_{G}$ such that $k$ is a nonnegative integer, $0 \leq$ $\beta<1, \delta_{G}>0$ and for all $|w|_{k, \beta} \leq \delta_{G}, \mathcal{G}^{\prime}[w] \in C^{k, \beta}(\bar{U})$. In addition, $\mathcal{G}^{\prime}$ is a (local) Lipschitz operator in the sense that for all $\left|w_{1}\right|_{k, \beta},\left|w_{2}\right|_{k, \beta} \leq \delta_{G}$, we have

$$
\left|\mathcal{G}^{\prime}\left[w_{1}\right]-\mathcal{G}^{\prime}\left[w_{2}\right]\right|_{k, \beta} \leq \kappa_{G}\left|w_{1}-w_{2}\right|_{k, \beta}
$$

A2. There exists $x \in \bar{U}$ such that $\mathcal{G}^{\prime}[0](x) \neq 0$.

A3. The Gaussian random field $\{\xi(x): x \in U\}$ belongs to the space $C^{k, \beta}(\bar{U})$ almost surely, that is, $\mathbb{P}\left(|\xi|_{k, \beta}<\infty\right)=1$. The covariance function $C(\cdot, \cdot)$ is positive definite and satisfies $\sup _{y \in \bar{U}}|C(\cdot, y)|_{k, 2 \beta}<\infty$. Moreover, we assume that $\sup _{y \in \bar{U}}\left|C_{D^{\gamma} \xi}(\cdot, y)\right|_{2 \beta}<\infty$ for all $\gamma$ such that $|\gamma| \leq k$, where we define

$$
C_{D^{\gamma} \xi}(x, y) \triangleq \mathbb{E}\left\{D^{\gamma} \xi(x) D^{\gamma} \xi(y)\right\} .
$$

With the above assumptions, we have the following sharp asymptotic approximation for the tail probability of $\omega(\sigma)$.

THEOREM 2. Under Assumptions A1-A3, for $0<\alpha<1$, we have

$$
\mathbb{P}\left\{\mathcal{G}(\sigma \xi)>\sigma^{\alpha}\right\}=\left(c_{2}+o(1)\right) \sigma^{1-\alpha} \exp \left(-\frac{1}{2} K_{\sigma}^{*}\right) \quad \text { as } \sigma \rightarrow 0,
$$

where $c_{2}=\left\{(2 \pi)^{-1} \mathcal{K}\left(\mathcal{G}^{\prime}[\mathbf{0}]\right)\right\}^{\frac{1}{2}}$ and

$$
K_{\sigma}^{*}=\min _{w \in \mathcal{B}, \mathcal{G}(\sigma \mathbf{C} w)=\sigma^{\alpha}} \mathcal{K}(w),
$$

where $\mathcal{K}$ is defined as in (12) and $\mathcal{B}$ is defined as in (13) with the same $\beta$ and $k$ as in Assumptions A1-A3.

The constants $k$ and $\beta$ in Assumptions A1-A3 are problem-dependent. For example, Li, Liu and $\mathrm{Xu}(2016)$ consider the functional $\mathcal{G}(\xi)=\int_{U} e^{\sigma \xi(t)+\mu(t)} d t-$ $\int_{U} e^{\mu(t)} d t$, where $\mu(\cdot) \in C(\bar{U})$ is a deterministic function. This particular $\mathcal{G}$ satisfies Assumptions A1 and A2 with $k=0$ and $\beta=0$.

Now we proceed to characterizing the solution to the optimization (11).

THEOREM 3. Under Assumptions A1-A3,

(i) the optimization problem (11) has a unique solution for $\sigma$ sufficiently small, denoted by $\xi^{*}$; 
(ii) we have the following approximation as $\sigma \rightarrow 0$

$$
\xi^{*}=\left(1+o_{k, \beta}(1)\right) \sigma^{\alpha-1} \frac{\mathcal{G}^{\prime}[\mathbf{0}]}{\mathcal{K}\left(\mathcal{G}^{\prime}[\mathbf{0}]\right)},
$$

where we write $h_{\sigma}(\cdot)=o_{k, \beta}(1)$ if $\left|h_{\sigma}\right|_{k, \beta}=o(1)$ as $\sigma \rightarrow 0$.

Combining Theorem 2 and Theorem 3, we arrive at the following approximation.

COROllary 1. Under the Assumptions A1-A3, for $0<\alpha<1$, we have

$$
\log \mathbb{P}\left\{\mathcal{G}(\sigma \xi)>\sigma^{\alpha}\right\}=-(1+o(1)) \frac{\sigma^{2 \alpha-2}}{2 \mathcal{K}\left(\mathcal{G}^{\prime}[\mathbf{0}]\right)}
$$

Intuitively, $\mathcal{G}(\sigma \xi)-\mathcal{G}(\mathbf{0}) \approx \sigma \int_{U} \mathcal{G}^{\prime}[\mathbf{0}](x) \xi(x) d x$ according to the first-order approximation of $\mathcal{G}$. Thus, the above corollary serves as a rigorous justification for the approximation

$$
\log \mathbb{P}\left\{\mathcal{G}(\sigma \xi)>\sigma^{\alpha}\right\} \approx \log \mathbb{P}\left[\sigma \int_{U} \mathcal{G}^{\prime}[\mathbf{0}](x) \xi(x) d x>\sigma^{\alpha}\right] \approx-\frac{\sigma^{2 \alpha-2}}{2 \mathcal{K}\left(\mathcal{G}^{\prime}[\mathbf{0}]\right)}
$$

If we are only interested in obtaining Corollary 1 , it may be possible to rely on the above heuristic and simplify the proof. However, if we would like to obtain a sharp asymptotic approximation as shown in Theorem 2, the first-order approximation is insufficient for obtaining the subexponential terms. To see this, we consider a simple example where $U=[0,1], \xi$ is a centered Gaussian random field living on $U$ with $\operatorname{Var}\left(\int_{0}^{1} \xi(x) d x\right)=1, \mathcal{G}(\sigma \xi)=e^{\sigma \int_{0}^{1} \xi(x) d x}-1$. Then

$$
\mathbb{P}\left\{\mathcal{G}(\sigma \xi)>\sigma^{\alpha}\right\}=\mathbb{P}\left(e^{\sigma Z}-1>\sigma^{\alpha}\right) \quad \text { and } \quad \sigma \int_{U} \mathcal{G}^{\prime}[\mathbf{0}](x) \xi(x) d x=\sigma Z,
$$

where $Z$ a standard Gaussian variable. With some careful calculations, it is not hard to show that

$$
\mathbb{P}\left(e^{\sigma Z}-1>\sigma^{\alpha}\right)=(1+o(1)) \mathbb{P}\left(\sigma Z>\sigma^{\alpha}\right)
$$

if and only if $\frac{2}{3}<\alpha<1$. Thus, for $0<\alpha<\frac{2}{3}$, we cannot prove Theorem 2 by simply employing the first-order approximation.

2.5. Numerical approximation. In this section, we present a numerical method, more precisely, an iterative algorithm, for computing the solution $\xi^{*}$ to (11). To solve the optimization, we introduce the Lagrangian multiplier $\lambda \in \mathbb{R}$ and define the Lagrangian function $L$ :

$$
L(\xi)=\iint \xi(x) C(x, y) \xi(y) d x d y-2 \frac{\lambda}{\sigma}\left(\mathcal{G}(\sigma \mathbf{C} \xi)-\sigma^{\alpha}\right) .
$$


The first-order condition $\frac{\partial L}{\partial \xi} \equiv 0$ implies the KKT condition for $\lambda$ and $\xi$ :

$$
\mathbf{C} \xi=\lambda \mathbf{C G}^{\prime}[\sigma \mathbf{C} \xi]
$$

Since the covariance function $C(x, y)$ is positive definite, and thus the linear map $\mathbf{C}$ is a bijection. The above condition becomes

$$
\xi=\lambda \mathcal{G}^{\prime}[\sigma \mathbf{C} \xi]
$$

The solution $\left(\xi^{*}, \lambda^{*}\right)$ to the constrained optimization problem is determined by

$$
\begin{aligned}
& \xi^{*}=\lambda^{*} \mathcal{G}^{\prime}\left[\sigma \mathbf{C} \xi^{*}\right], \\
& \mathcal{G}\left(\sigma \mathbf{C} \xi^{*}\right)=\sigma^{\alpha} .
\end{aligned}
$$

Our strategy is to first find $\lambda$ given $\xi$ to satisfy the constraint (16b); and then we look for $\xi$ and the corresponding $\lambda=\Lambda(\xi)$ determined by the previous step to satisfy the fix point equation (16a). Motivated by this, we define a functional

$$
\Lambda: \mathcal{B} \rightarrow\left[-\sigma^{\alpha-1-\varepsilon}, \sigma^{\alpha-1-\varepsilon}\right]
$$

such that for each $w \in \mathcal{B}, \lambda=\Lambda(w)$ solves the following equation:

$$
\mathcal{G}\left(\sigma \mathbf{C} \lambda \mathcal{G}^{\prime}[\sigma \mathbf{C} w]\right)=\sigma^{\alpha} .
$$

To see that $\Lambda(\cdot)$ is well-defined, for each $w \in \mathcal{B}$ we define the function $T_{w}$ : $\left[-\sigma^{\alpha-1-\varepsilon}, \sigma^{\alpha-1-\varepsilon}\right] \rightarrow \mathbb{R}$

$$
T_{w}(\lambda)=\lambda-\mathcal{K}\left(\mathcal{G}^{\prime}[\mathbf{0}]\right)^{-1} \sigma^{-1}\left(\mathcal{G}\left(\sigma \mathbf{C} \lambda \mathcal{G}^{\prime}[\sigma \mathbf{C} w]\right)-\sigma^{\alpha}\right) .
$$

Clearly, solutions to (17) are fixed points of the function $T_{w}(\cdot)$. The well-posedness of the function $\Lambda(\cdot)$ is then established by the next proposition.

Proposition 1. For $\sigma$ sufficiently small, $w \in \mathcal{B}$, and $\left|\lambda_{1}\right|,\left|\lambda_{2}\right| \leq \sigma^{\alpha-1-\varepsilon}$, we have that $\left|T_{w}\left(\lambda_{1}\right)\right|,\left|T_{w}\left(\lambda_{2}\right)\right| \leq \sigma^{\alpha-1-\varepsilon}$ and there exists a constant $\kappa_{T}$ independent of $\sigma$ and $w$, such that

$$
\left|T_{w}\left(\lambda_{1}\right)-T_{w}\left(\lambda_{2}\right)\right| \leq \kappa_{T} \sigma^{\alpha-\varepsilon}\left|\lambda_{1}-\lambda_{2}\right|
$$

The above proposition and the contraction mapping theorem guarantee that for each $w \in \mathcal{B}, T_{w}(\cdot)$ has a unique fixed point in $\left[-\sigma^{\alpha-1-\varepsilon}, \sigma^{\alpha-1-\varepsilon}\right]$. Therefore, there is a unique solution $\Lambda(w) \in\left[-\sigma^{\alpha-1-\varepsilon}, \sigma^{\alpha-1-\varepsilon}\right]$ satisfying (17). Furthermore, it ensures the convergence of the iterative algorithm based on the contraction mapping $T_{w}(\lambda)$. We further define an operator $\Xi$.

$$
\Xi[w]=\Lambda(w) \mathcal{G}^{\prime}[\sigma \mathbf{C} w] .
$$

The solution $\xi^{*}$ to (11) and equivalently (16) is a fixed point of $\Xi$. 
PROPOSITION 2. For $\sigma$ sufficiently small, $\Xi$ is a contraction mapping over $\mathcal{B}$. More specifically, there exists a constant $\kappa_{\Xi}$ such that for all $w_{1}, w_{2} \in \mathcal{B}$, we have

$$
\left|\Xi\left[w_{1}\right]-\Xi\left[w_{2}\right]\right|_{k, \beta} \leq \kappa \Xi \sigma^{\alpha}\left|w_{1}-w_{2}\right|_{k, \beta}
$$

The above proposition and the contraction mapping theorem guarantee that (16) has a unique solution that is $\left(\lambda^{*}, \xi^{*}\right)$ in $\left[-\sigma^{\alpha-1-\varepsilon}, \sigma^{\alpha-1-\varepsilon}\right] \times \mathcal{B}$. Furthermore, this solution can be computed numerically via the following iterative algorithm:

1. Initialize $\hat{\xi}_{0}^{*}=\sigma^{\alpha-1} \frac{\mathcal{G}^{\prime}[\mathbf{0}]}{\mathcal{K}\left(\mathcal{G}^{\prime}[\mathbf{0}]\right)}$.

2. At $l$ th iteration, update $\hat{\xi}_{l}^{*}$ by

$$
\hat{\xi}_{l}^{*}=\Xi\left[\hat{\xi}_{l-1}^{*}\right] .
$$

According to the contraction mapping theorem, the rate of convergence is

$$
\left|\hat{\xi}_{l}^{*}-\xi^{*}\right|_{k, \beta} \leq\left(\kappa_{\Xi} \sigma^{\alpha}\right)^{l}\left|\hat{\xi}_{0}^{*}-\xi^{*}\right|_{k, \beta}=O\left(\sigma^{\alpha l+\alpha-1}\right) .
$$

Therefore, if we run $l>\frac{2(1-\alpha)}{\alpha}$ iterations, then $\left|\hat{\xi}_{l}^{*}-\xi^{*}\right|_{k, \beta}=o\left(\sigma^{1-\alpha}\right)$, and we could use $\mathcal{K}\left(\hat{\xi}_{l}^{*}\right)$ to approximate $K_{\sigma}^{*}$ in Theorem 2 .

3. Technical proofs. Throughout the proof, we will use $\kappa_{0}$ as generic notation for large and not-so-important constants whose value may vary from place to place. Similarly, we use $\varepsilon_{0}$ as generic notation for small positive constants. Furthermore, for two sequences $a_{\sigma}$ and $b_{\sigma}$, we write $a_{\sigma}=o\left(b_{\sigma}\right)$ if $b_{\sigma} / a_{\sigma} \rightarrow 0$ as $\sigma$ tend to zero and $a_{\sigma}=O\left(b_{\sigma}\right)$ if $b_{\sigma} / a_{\sigma}$ is bounded when $\sigma$ varies. Moreover, for two sequences of functions $a_{\sigma}(\cdot)$ and $b_{\sigma}(\cdot)$, we write $a_{\sigma}=o_{k, \beta}\left(b_{\sigma}\right)$ if $\left|a_{\sigma}\right|_{k, \beta}=o\left(\left|b_{\sigma}\right|_{k, \beta}\right)$ and $a_{\sigma}=O_{k, \beta}\left(b_{\sigma}\right)$ if $\left|a_{\sigma}\right|_{k, \beta}=O\left(\left|b_{\sigma}\right|_{k, \beta}\right)$.

The proofs in this sections are organized as follows. The proof of Theorem 2 is presented in Section 3.1. Section 3.2 presents proofs of Propositions 1, 2 and Theorem 3. Section 3.3 shows the proof of Theorem 1. The proofs of supporting lemmas are postponed to the Appendix.

3.1. Proof of Theorem 2. We start with a useful lemma that restrict our analysis on the event $\mathcal{L}=\left\{\xi-\mathbf{C} \xi^{*} \in \mathcal{B}\right\}$, whose proof will be presented in Section 3.3.

LEMMA 1. There exists a positive constant $\varepsilon_{0}$ such that

$$
\mathbb{P}\left(\xi-\mathbf{C} \xi^{*} \in \mathcal{B}^{c}\right) \leq e^{-\varepsilon_{0} \sigma^{2 \alpha-2-2 \varepsilon}} .
$$

Proof For Theorem 2. Let $\xi^{*}$ be the solution to (11). We define an exponential change of measure:

$$
\frac{d \mathbb{Q}}{d \mathbb{P}}=\exp \left(\int_{U} \xi^{*}(x) \xi(x) d x-\frac{1}{2} \int_{U} \int_{U} \xi^{*}(x) C(x, y) \xi^{*}(y) d x d y\right) .
$$


Under measure $\mathbb{Q}, \xi(x)$ is a Gaussian random field with mean function $\mathbf{C} \xi^{*}(x)$ and covariance function $C(x, y)$. Let

$$
\mathcal{L}=\left\{\xi-\mathbf{C} \xi^{*} \in \mathcal{B}\right\} .
$$

According to Lemma 1, we only need to consider the event restricted to $\mathcal{L}$. By means of the change of measure $\mathbb{Q}$, we have

$$
\begin{aligned}
\mathbb{P}\left(\mathcal{G}(\sigma \xi)>\sigma^{\alpha}, \mathcal{L}\right) \\
=\mathbf{E}^{\mathbb{Q}}\left[\frac{d \mathbb{P}}{d \mathbb{Q}} ; \mathcal{G}(\sigma \xi)>\sigma^{\alpha}, \mathcal{L}\right] \\
=\exp \left(\frac{1}{2} \int_{U \times U} \xi^{*}(x) C(x, y) \xi^{*}(y) d x d y\right) \\
\quad \times \mathbf{E}^{\mathbb{Q}}\left[e^{-\int_{U} \xi^{*}(x) \xi(x) d x} ; \mathcal{G}(\sigma \xi)>\sigma^{\alpha}, \mathcal{L}\right],
\end{aligned}
$$

where $\mathbf{E}^{\mathbb{Q}}$ denotes the expectation with respect to the measure $\mathbb{Q}$. It is easy to check that the random field $\mathbf{C} \xi^{*}(x)+\xi(x)$ under $\mathbb{P}$ has the same distribution as $\xi(x)$ under $\mathbb{Q}$. Thus, we replace the probability measure $\mathbb{Q}$ and $\xi$ with $\mathbb{P}$ and $\mathbf{C} \xi^{*}+\xi$ in (20) and obtain

$$
\begin{aligned}
& \mathbb{P}\left(\mathcal{G}(\sigma \xi)>\sigma^{\alpha}, \mathcal{L}\right) \\
&=\exp \left(\frac{1}{2} \int_{U \times U} \xi^{*}(x) C(x, y) \xi^{*}(y) d x d y\right) \\
& \times \mathbf{E}\left[e^{-\int_{U} \xi^{*}(x)\left(\mathbf{C} \xi^{*}(x)+\xi(x)\right) d x} ; \mathcal{G}\left(\sigma\left(\xi+\mathbf{C} \xi^{*}\right)\right)>\sigma^{\alpha}, \xi \in \mathcal{B}\right] \\
&=\exp \left(-\frac{1}{2} \int_{U \times U} \xi^{*}(x) C(x, y) \xi^{*}(y) d x d y\right) \\
& \times \mathbf{E}\left[e^{-\int_{U} \xi^{*}(x) \xi(x) d x} ; \mathcal{G}\left(\sigma\left(\xi+\mathbf{C} \xi^{*}\right)\right)-\mathcal{G}\left(\sigma \mathbf{C} \xi^{*}\right)>0, \xi \in \mathcal{B}\right] \\
&= e^{-\frac{1}{2} K_{\sigma}^{*}} \times \mathbf{E}\left[e^{-\int_{U} \xi^{*}(x) \xi(x) d x} ; \mathcal{G}\left(\sigma\left(\xi+\mathbf{C} \xi^{*}\right)\right)-\mathcal{G}\left(\sigma \mathbf{C} \xi^{*}\right)>0, \xi \in \mathcal{B}\right] .
\end{aligned}
$$

We define two events

$$
\begin{aligned}
F & =\left\{\mathcal{G}\left(\sigma\left(\xi+\mathbf{C} \xi^{*}\right)\right)-\mathcal{G}\left(\sigma \mathbf{C} \xi^{*}\right)>0\right\} \quad \text { and } \\
F_{1} & =\left\{\int_{U} \mathcal{G}^{\prime}\left[\sigma \mathbf{C} \xi^{*}\right](x) \sigma \xi(x) d x>0\right\} .
\end{aligned}
$$

Let the event $\mathcal{L}_{1}=\{\xi \in \mathcal{B}\}$. We will present an approximation for

$$
I_{1}=\mathbf{E}\left[e^{-\int_{U} \xi^{*}(x) \xi(x) d x} ; F_{1}\right]
$$

and show that

$$
I_{2}=\mathbf{E}\left[e^{-\int_{U} \xi^{*}(x) \xi(x) d x} ;\left(F_{1} \triangle F\right) \cap \mathcal{L}_{1}\right]
$$


is ignorable, where " $\triangle$ " denotes the symmetric difference between two sets. First, we compute

$$
I_{1}=\mathbf{E}\left[e^{-\int_{U} \xi^{*}(x) \xi(x) d x} ; \int_{U} \mathcal{G}^{\prime}\left[\sigma \mathbf{C} \xi^{*}\right](x) \xi(x) d x>0\right] .
$$

According to Proposition 2 whose proof is independent of the current one, $\xi^{*}$ is the fixed point of the contraction map $\Xi$, and thus

$$
\xi^{*}=\Xi\left[\xi^{*}\right]=\Lambda\left(\xi^{*}\right) \mathcal{G}^{\prime}\left[\sigma \mathbf{C} \xi^{*}\right]
$$

Therefore, $\xi^{*}$ and $\mathcal{G}^{\prime}\left[\sigma \mathbf{C} \xi^{*}\right]$ are different only by a factor of $\Lambda\left(\xi^{*}\right)$. Thus, $\int_{U} \xi^{*}(x) \xi(x) d x$ and $\int_{U} \mathcal{G}^{\prime}\left[\sigma \mathbf{C} \xi^{*}\right](x) \xi(x) d x>0$ are different by a factor $\Lambda\left(\xi^{*}\right)$. The following lemma establishes an approximation for $\Lambda\left(\xi^{*}\right)$.

LEMMA 2. For all $w \in \mathcal{B}, \Lambda(w)=\mathcal{K}\left(\mathcal{G}^{\prime}[\mathbf{0}]\right)^{-1} \sigma^{\alpha-1}(1+o(1))$. This approximation is uniform in $w$.

Thanks to Lemma 2, we have

$$
\Lambda\left(\xi^{*}\right)=(1+o(1)) \frac{\kappa \sigma^{\alpha-1}}{\mathcal{K}\left(\mathcal{G}^{\prime}[\mathbf{0}]\right)} .
$$

Let $Z_{1}=\int_{U} \xi^{*}(x) \xi(x) d x$, then $Z_{1}$ is a normally distributed random variable with a zero mean. The expectation (21) can be computed as follows:

$$
\begin{aligned}
& \mathbf{E}\left[e^{-Z_{1}} ; Z_{1}>0\right] \\
& \quad=\int_{0}^{\infty} \frac{1}{\sqrt{2 \pi \operatorname{Var}\left(Z_{1}\right)}} e^{-\frac{z_{1}^{2}}{2 \operatorname{Var}\left(Z_{1}\right)}-z_{1}} d z_{1} \\
& \quad=\frac{1}{\sqrt{2 \pi \operatorname{Var}\left(Z_{1}\right)}} E\left[e^{\left.-\frac{V^{2}}{2 \operatorname{Var}\left(Z_{1}\right)}\right]},\right.
\end{aligned}
$$

where $V$ is a random variable following the exponential distribution with rate 1 . Notice that

$$
\operatorname{Var}\left(Z_{1}\right)=\int_{U \times U} \xi^{*}(x) C(x, y) \xi^{*}(y) d x d y=(1+o(1)) \sigma^{2 \alpha-2} \mathcal{K}^{-1}\left[\mathcal{G}^{\prime}[\mathbf{0}]\right]
$$

The second equality is obtained with the aid of Theorem 3(ii). The above display, (22) and dominated convergence theorem give

$$
I_{1}=\left\{(2 \pi)^{-1} \mathcal{K}\left(\mathcal{G}^{\prime}[\mathbf{0}]\right)\right\}^{1 / 2} \sigma^{1-\alpha}(1+o(1)) .
$$

Now, we proceed to the term $I_{2}$.

LEMMA 3. Under Assumption A1, we have that for $\left|w_{1}\right|_{k, \beta},\left|w_{2}\right|_{k, \beta} \leq \delta_{G}$, $\left|w_{1}-w_{2}\right|_{k, \beta}^{-2}\left|\mathcal{G}\left(w_{1}\right)-\mathcal{G}\left(w_{2}\right)-\int_{U} \mathcal{G}^{\prime}\left[w_{2}\right](x)\left(w_{1}(x)-w_{2}(x)\right) d x\right| \leq \operatorname{meas}(U) \kappa_{G}$, where meas $(U)$ is the Lebesgue measure of $U$ and $k, \beta, \delta_{G}, \kappa_{G}$ are constants appeared in Assumption A1. 
According to Lemma 3, we have that for $\sigma$ sufficiently small and $\xi \in \mathcal{B}$,

$$
\begin{aligned}
\left|\mathcal{G}\left(\sigma\left(\xi+\mathbf{C} \xi^{*}\right)\right)-\mathcal{G}\left(\sigma \mathbf{C} \xi^{*}\right)-\sigma \int_{U} \mathcal{G}^{\prime}\left[\sigma \mathbf{C} \xi^{*}\right](x) \xi(x) d x\right| \\
\leq \operatorname{meas}(U) \kappa_{G} \sigma^{2}|\xi|_{k, \beta}^{2} .
\end{aligned}
$$

Note that on the event $F_{1} \triangle F, \mathcal{G}\left(\sigma\left(\xi+\mathbf{C} \xi^{*}\right)\right)-\mathcal{G}\left(\sigma \mathbf{C} \xi^{*}\right)$ and $\sigma \int_{U} \mathcal{G}^{\prime}\left[\sigma \mathbf{C} \xi^{*}\right](x) \xi(x) d x$ have opposite signs, and thus

$$
\begin{aligned}
& \left|\mathcal{G}\left(\sigma\left(\xi+\mathbf{C} \xi^{*}\right)\right)-\mathcal{G}\left(\sigma \mathbf{C} \xi^{*}\right)-\sigma \int_{U} \mathcal{G}^{\prime}\left[\sigma \mathbf{C} \xi^{*}\right](x) \xi(x) d x\right| \\
& \quad \geq\left|\sigma \int_{U} \mathcal{G}^{\prime}\left[\sigma \mathbf{C} \xi^{*}\right](x) \xi(x) d x\right| .
\end{aligned}
$$

We combine (24) and (25) and arrive at

$$
\left(F \triangle F_{1}\right) \cap \mathcal{L}_{1} \subset\left\{\operatorname{meas}(U) \kappa_{G}\|\xi\|_{k, \beta}^{2} \geq \sigma^{-1}\left|\int_{U} \mathcal{G}^{\prime}\left[\sigma \mathbf{C}^{*}\right](x) \xi(x) d x\right|\right\} \cap \mathcal{L}_{1} .
$$

We write $Z_{2}=\|\xi\|_{k, \beta}^{2}$, then the above display implies that

$$
\left(F \triangle F_{1}\right) \cap \mathcal{L}_{1} \subset\left\{\operatorname{meas}(U) \kappa_{G} Z_{2} \geq \sigma^{-1} \Lambda\left(\xi^{*}\right)^{-1}\left|Z_{1}\right|\right\} \cap \mathcal{L}_{1} .
$$

This gives an upper bound of the expectation

$$
\mathbf{E}\left[e^{-\int_{U} \xi^{*}(x) \xi(x) d x} ;\left(F \triangle F_{1}\right) \cap \mathcal{L}_{1}\right] \leq \mathbf{E}\left[e^{-Z_{1}} ; \kappa_{G} Z_{2} \geq \sigma^{-1} \Lambda\left(\xi^{*}\right)^{-1}\left|Z_{1}\right|, \mathcal{L}_{1}\right] .
$$

On the event $\left\{0<\left|Z_{1}\right| \leq \sigma^{\varepsilon}\right\}$, this expectation is negligible compared to $I_{1}$, that is,

$$
\mathbf{E}\left[e^{Z_{1}} ; 0<\left|Z_{1}\right|<\sigma^{\varepsilon}\right]=O\left(\mathbb{P}\left(0<\left|Z_{1}\right|<\sigma^{\varepsilon}\right)\right)=O\left(\sigma^{1-\alpha+\varepsilon}\right) .
$$

The second equality in the above display is due to (23). Furthermore, on the set $\mathcal{L}_{1}$, we have $\left|Z_{1}\right| \leq\left|\xi^{*}\right|_{0}|\xi|_{0} \kappa_{0} \leq \kappa_{0} \sigma^{2 \alpha-2-\varepsilon}$, where $\kappa_{0}$ is a sufficiently large constant. Therefore, we only need to focus on the expectation

$$
\begin{aligned}
& \mathbf{E}\left[e^{Z_{1}} ; \sigma^{\varepsilon}<\left|Z_{1}\right|<\kappa_{0} \sigma^{2 \alpha-2-\varepsilon}, Z_{2}>\Lambda\left(\xi^{*}\right)^{-1}\left|Z_{1} / \sigma\right|\right] \\
& =\int_{\sigma^{\varepsilon}}^{\kappa_{0} \sigma^{2 \alpha-2-\varepsilon}} e^{z} \mathbb{P}\left(Z_{2}>\Lambda\left(\xi^{*}\right)^{-1} z / \sigma \mid Z_{1}=z\right) p_{Z_{1}}(z) d z \\
& \quad+\int_{\sigma^{\varepsilon}}^{\kappa_{0} \sigma^{2 \alpha-2-\varepsilon}} e^{z} \mathbb{P}\left(Z_{2}>\Lambda\left(\xi^{*}\right)^{-1} z / \sigma \mid Z_{1}=-z\right) p_{Z_{1}}(z) d z,
\end{aligned}
$$

where $p_{Z_{1}}(z)$ is the density function of $Z_{1}$.

LEMMA 4. For $z \in\left[\sigma^{\varepsilon}, \kappa_{0} \sigma^{2 \alpha-2-\varepsilon}\right]$, there exists a constant $\varepsilon_{0}>0$ such that

$$
\begin{aligned}
\mathbb{P}\left(Z_{2}\right. & \left.>\Lambda\left(\xi^{*}\right)^{-1} z / \sigma \mid Z_{1}=z\right)+\mathbb{P}\left(Z_{2}>\Lambda\left(\xi^{*}\right)^{-1} z / \sigma \mid Z_{1}=-z\right) \\
& \leq e^{-\varepsilon_{0} \sigma^{-\alpha} z} .
\end{aligned}
$$


With the above lemma, the expectation (27) is bounded by

$$
\begin{aligned}
(27) & \leq \int_{\sigma^{\varepsilon}}^{\kappa_{0} \sigma^{2 \alpha-2-\varepsilon}} e^{-\left(\varepsilon_{0} \sigma^{-\alpha}-1\right) z} p_{Z_{1}}(z) d z \\
& =\frac{1}{\sqrt{2 \pi \operatorname{Var}\left(Z_{1}\right)}} \int_{\sigma^{\varepsilon}}^{\kappa_{0} \sigma^{2 \alpha-2-\varepsilon}} e^{-\left(\varepsilon_{0} \sigma^{-\alpha}-1\right) z-\frac{z^{2}}{2 \operatorname{Var}\left(Z_{1}\right)}} d z \\
& \leq \frac{1}{\sqrt{2 \pi \operatorname{Var}\left(Z_{1}\right)}} \int_{\sigma^{\varepsilon}}^{\kappa_{0} \sigma^{2 \alpha-2-\varepsilon}} e^{-\frac{\varepsilon_{0}}{2} \sigma^{-\alpha} z} d z,
\end{aligned}
$$

for $\sigma$ sufficiently small so that $\varepsilon_{0} \sigma^{-\alpha}-1>\frac{\varepsilon_{0}}{2} \sigma^{-\alpha}$. The above inequality is further bounded by

$$
\begin{aligned}
(27) & \leq \frac{1}{\sqrt{2 \pi \operatorname{Var}\left(Z_{1}\right)}} \int_{\sigma^{\varepsilon}}^{\kappa_{0} \sigma^{2 \alpha-2-\varepsilon}} e^{-\frac{\varepsilon_{0}}{2} \sigma^{-\alpha} z} d z \\
& \leq \frac{1}{\sqrt{2 \pi \operatorname{Var}\left(Z_{1}\right)}} \kappa_{0} \sigma^{2 \alpha-2-\varepsilon} e^{-\frac{\varepsilon_{0}}{2} \sigma^{-\alpha+\varepsilon}} \\
& =O\left(e^{-\frac{\varepsilon_{0}}{2} \sigma^{-\alpha+\varepsilon}}\right) .
\end{aligned}
$$

Therefore,

$$
(27)=o\left(\sigma^{1-\alpha}\right) \text {. }
$$

We combine our analysis for $I_{1}$ and $I_{2}$ and conclude our proof for Theorem 2 .

\subsection{Proofs of Proposition 1, 2, and Theorem 3.}

Proof of Proposition 1. Note that as $\sigma$ tends to zero, we have $\sigma \mathbf{C} w=$ $o_{k, \beta}(1), \mathcal{G}^{\prime}[\sigma \mathbf{C} w]=\mathcal{G}^{\prime}[\mathbf{0}]+o_{k, \beta}(1)$ and $\sigma \mathbf{C} \lambda \mathcal{G}^{\prime}[\sigma \mathbf{C} w]=o_{k, \beta}(1)$ for all $|\lambda| \leq$ $\sigma^{\alpha-1-\varepsilon}$ and $w \in \mathcal{B}$. This allows us to expand $\mathcal{G}\left(\sigma \mathbf{C} \lambda \mathcal{G}^{\prime}[\sigma \mathbf{C} w]\right)$ near the origin. We elaborate this expansion as follows. First, according to Assumption A1, we have that there exists a constant $\varepsilon_{0}$ such that for all $w \in \mathcal{B}$ and $\sigma \leq \varepsilon_{0}$,

$$
\mathcal{G}^{\prime}[\sigma \mathbf{C} w]=\mathcal{G}^{\prime}[\mathbf{0}]+O_{k, \beta}(\sigma \mathbf{C} w) .
$$

Second, with the aid of (29) we have that for all $\left|\lambda_{1}\right|,\left|\lambda_{2}\right| \leq \sigma^{\alpha-1-\varepsilon}$ and $w \in \mathcal{B}$,

$$
\sigma \mathbf{C} \lambda_{1} \mathcal{G}^{\prime}[\sigma \mathbf{C} w]-\sigma \mathbf{C} \lambda_{2} \mathcal{G}^{\prime}[\sigma \mathbf{C} w]=\sigma\left(\lambda_{1}-\lambda_{2}\right) \mathbf{C}\left\{\mathcal{G}^{\prime}[\mathbf{0}]+O_{k, \beta}(\sigma \mathbf{C} w)\right\} .
$$

Thanks to Lemma 3 on page 2792 and (30), we have that for all $\left|\lambda_{1}\right|,\left|\lambda_{2}\right| \leq \sigma^{\alpha-1-\varepsilon}$ and $w \in \mathcal{B}$,

$$
\begin{aligned}
& \mathcal{G}\left(\sigma \mathbf{C} \lambda_{1} \mathcal{G}^{\prime}[\sigma \mathbf{C} w]\right)-\mathcal{G}\left(\sigma \mathbf{C} \lambda_{2} \mathcal{G}^{\prime}[\sigma \mathbf{C} w]\right) \\
& \quad=\int_{U} \mathcal{G}^{\prime}\left[\sigma \mathbf{C} \lambda_{2} \mathcal{G}^{\prime}[\sigma \mathbf{C} w]\right](x) v(x) d x+O\left(|v|_{k, \beta}^{2}\right),
\end{aligned}
$$


where we define

$$
v(x)=\sigma \mathbf{C} \lambda_{1} \mathcal{G}^{\prime}[\sigma \mathbf{C} w](x)-\sigma \mathbf{C} \lambda_{2} \mathcal{G}^{\prime}[\sigma \mathbf{C} w](x) .
$$

Setting $w$ as $\lambda_{2} \mathcal{G}^{\prime}[\sigma \mathbf{C} w]$ in (29), we have

$$
\begin{aligned}
\mathcal{G}^{\prime}\left[\sigma \mathbf{C} \lambda_{2} \mathcal{G}^{\prime}[\sigma \mathbf{C} w]\right] & =\mathcal{G}^{\prime}[\mathbf{0}]+O_{k, \beta}\left(\sigma \mathbf{C} \lambda_{2} \mathcal{G}^{\prime}[\sigma \mathbf{C} w]\right) \\
& =\mathcal{G}^{\prime}[\mathbf{0}]+O_{k, \beta}\left(\sigma \lambda_{2} \mathcal{G}^{\prime}[\mathbf{0}]\right) .
\end{aligned}
$$

The last equality in the above display is due to (29) and the fact $O_{k, \beta}(\sigma \mathbf{C w})=$ $o_{k, \beta}(1)$. According to (30) and (32), we have

$$
\begin{aligned}
& \int_{U} \mathcal{G}^{\prime}\left[\sigma \mathbf{C} \lambda_{2} \mathcal{G}^{\prime}[\sigma \mathbf{C} w]\right](x) v(x) d x \\
&= \sigma\left(\lambda_{1}-\lambda_{2}\right)\left\{\int_{U} \mathbf{C} \mathcal{G}^{\prime}[\mathbf{0}](x) \mathcal{G}^{\prime}[\mathbf{0}](x) d x\right. \\
&+O\left(\int_{U} \sigma^{2} \lambda_{2} \mathcal{G}^{\prime}[\mathbf{0}](x) \mathcal{G}^{\prime}(x) d x\right) \\
&+O\left(\int_{U} \sigma \mathcal{G}^{\prime}[\mathbf{0}](x) \mathbf{C} w(x) d x\right) \\
&\left.+O\left(\sigma \lambda_{2} \sigma \int_{U} \mathcal{G}^{\prime}[\mathbf{0}](x) \mathbf{C} w(x) d x\right)\right\} .
\end{aligned}
$$

Note that for $\lambda_{2} \in\left[-\sigma^{\alpha-1-\varepsilon}, \sigma^{\alpha-1-\varepsilon}\right]$ the above expression is simplified as

$$
\begin{aligned}
\int_{U} \mathcal{G}^{\prime} & {\left[\sigma \mathbf{C} \lambda_{2} \mathcal{G}^{\prime}[\sigma \mathbf{C} w]\right](x) v(x) d x } \\
& =\sigma\left(\lambda_{1}-\lambda_{2}\right)\left\{\int_{U} \mathbf{C} \mathcal{G}^{\prime}[\mathbf{0}](x) \mathcal{G}^{\prime}[\mathbf{0}](x) d x+O\left(\sigma^{\alpha-\varepsilon}\right)\right\} \\
& =\sigma\left(\lambda_{1}-\lambda_{2}\right)\left\{\mathcal{K}\left(\mathcal{G}^{\prime}[\mathbf{0}]\right)+O\left(\sigma^{\alpha-\varepsilon}\right)\right\} .
\end{aligned}
$$

Combining the above expression with (31), we have that for $\left|\lambda_{1}\right|,\left|\lambda_{2}\right| \leq \sigma^{\alpha-1-\varepsilon}$ and $w \in \mathcal{B}$,

$$
\begin{aligned}
& \mathcal{G}\left(\sigma \mathbf{C} \lambda_{1} \mathcal{G}^{\prime}[\sigma \mathbf{C} w]\right)-\mathcal{G}\left(\sigma \mathbf{C} \lambda_{2} \mathcal{G}^{\prime}[\sigma \mathbf{C} w]\right) \\
& =\sigma\left(\lambda_{1}-\lambda_{2}\right)\left\{\mathcal{K}\left(\mathcal{G}^{\prime}[\mathbf{0}]\right)+O\left(\sigma^{\alpha-\varepsilon}\right)\right\}+O\left(\sigma^{2}\left(\lambda_{1}-\lambda_{2}\right)^{2}\right),
\end{aligned}
$$

which can be simplified as

$$
\begin{array}{r}
\mathcal{G}\left(\sigma \mathbf{C} \lambda_{1} \mathcal{G}^{\prime}[\sigma \mathbf{C} w]\right)-\mathcal{G}\left(\sigma \mathbf{C} \lambda_{2} \mathcal{G}^{\prime}[\sigma \mathbf{C} w]\right) \\
=\sigma\left(\lambda_{1}-\lambda_{2}\right)\left\{\mathcal{K}\left(\mathcal{G}^{\prime}[\mathbf{0}]\right)+O\left(\sigma^{\alpha-\varepsilon}\right)\right\} .
\end{array}
$$

Recall the definition of $T_{w}(\lambda)$, we plug the above expression into the difference $T_{w}\left(\lambda_{1}\right)-T_{w}\left(\lambda_{2}\right)$, and arrive at

$T_{w}\left(\lambda_{1}\right)-T_{w}\left(\lambda_{2}\right)=\lambda_{1}-\lambda_{2}-\mathcal{K}\left(\mathcal{G}^{\prime}[\mathbf{0}]\right)^{-1} \sigma^{-1} \times \sigma\left(\lambda_{1}-\lambda_{2}\right)\left\{\mathcal{K}\left(\mathcal{G}^{\prime}[\mathbf{0}]\right)+O\left(\sigma^{\alpha-\varepsilon}\right)\right\}$, 
which is simplified as

$$
T_{w}\left(\lambda_{1}\right)-T_{w}\left(\lambda_{2}\right)=-\mathcal{K}\left(\mathcal{G}^{\prime}[\mathbf{0}]\right)^{-1}\left(\lambda_{1}-\lambda_{2}\right) \times O\left(\sigma^{\alpha-\varepsilon}\right) .
$$

The above expression implies that for $\left|\lambda_{1}\right|,\left|\lambda_{2}\right| \leq \sigma^{\alpha-1-\varepsilon}$,

$$
T_{w}\left(\lambda_{1}\right)-T_{w}\left(\lambda_{2}\right)=\left(\lambda_{1}-\lambda_{2}\right) \times O\left(\sigma^{\alpha-\varepsilon}\right) .
$$

This shows that $T_{w}(\lambda)$ is a contraction mapping for $\lambda \in\left[-\sigma^{\alpha-1-\varepsilon}, \sigma^{\alpha-1-\varepsilon}\right]$. To see $T_{w}(\lambda) \in\left[-\sigma^{\alpha-1-\varepsilon}, \sigma^{\alpha-1-\varepsilon}\right]$ for $\lambda \in\left[-\sigma^{\alpha-1-\varepsilon}, \sigma^{\alpha-1-\varepsilon}\right]$ and $w \in \mathcal{B}$, we let $\lambda_{2}=0$ and $\lambda_{1}=\lambda$ in (36) and obtain that

$$
T_{w}(\lambda)-T_{w}(0)=\lambda O\left(\sigma^{\alpha-\varepsilon}\right)=O\left(\sigma^{2 \alpha-1-2 \varepsilon}\right) .
$$

Recall that $T_{w}(0)=-\mathcal{K}\left(\mathcal{G}^{\prime}[\mathbf{0}]\right)^{-1} \sigma^{-1} \sigma^{\alpha}=-\kappa \mathcal{K}\left(\mathcal{G}^{\prime}[\mathbf{0}]\right)^{-1} \sigma^{\alpha-1}$. This implies

$$
T_{w}(\lambda)=\kappa \mathcal{K}\left(\mathcal{G}^{\prime}[\mathbf{0}]\right)^{-1} \sigma^{\alpha-1}(1+o(1)) \in\left[-\sigma^{\alpha-1-\varepsilon}, \sigma^{\alpha-1-\varepsilon}\right]
$$

and concludes our proof.

Proof of Proposition 2. According to the definition of $\Xi$,

$$
\Xi\left[w_{1}\right]-\Xi\left[w_{2}\right]=\Lambda\left(w_{1}\right)\left(\mathcal{G}^{\prime}\left[\sigma \mathbf{C} w_{1}\right]-\mathcal{G}^{\prime}\left[\sigma \mathbf{C} w_{2}\right]\right)+\left(\Lambda\left(w_{1}\right)-\Lambda\left(w_{2}\right)\right) \mathcal{G}^{\prime}\left[\sigma \mathbf{C} w_{2}\right]
$$

Therefore, we have

$$
\begin{aligned}
\mid \Xi\left[w_{1}\right] & -\left.\Xi\left[w_{2}\right]\right|_{k, \beta} \\
\leq & \left|\Lambda\left(w_{1}\right)\right| \times\left|\left(\mathcal{G}^{\prime}\left[\sigma \mathbf{C} w_{1}\right]-\mathcal{G}^{\prime}\left[\sigma \mathbf{C} w_{2}\right]\right)\right|_{k, \beta} \\
& +\left|\Lambda\left(w_{1}\right)-\Lambda\left(w_{2}\right)\right| \times\left|\mathcal{G}^{\prime}\left[\sigma \mathbf{C} w_{2}\right]\right|_{k, \beta} .
\end{aligned}
$$

We establish upper bound for the first and second terms on the right-hand side of the above inequality separately. To start with, according to Assumptions A1 and A3 that $\sup _{y \in \bar{U}}|C(\cdot, y)|_{k, 2 \beta}<\infty$, for $w_{1}, w_{2} \in \mathcal{B}$, we have

$$
\begin{aligned}
& \left|\Lambda\left(w_{1}\right)\right| \times\left|\left(\mathcal{G}^{\prime}\left[\sigma \mathbf{C} w_{1}\right]-\mathcal{G}^{\prime}\left[\sigma \mathbf{C} w_{2}\right]\right)\right|_{k, \beta} \\
& =O\left(\sigma\left|\Lambda\left(w_{1}\right)\right|\left|w_{1}-w_{2}\right|_{k, \beta}\right)=O\left(\sigma^{\alpha}\right)\left|w_{1}-w_{2}\right|_{k, \beta} .
\end{aligned}
$$

The second equality in the above expression is due to Lemma 2 on page 2792 . We proceed to the second term on the right-hand side of (38). Because $\Lambda(w)$ is the fixed point of $T_{w}(\cdot)$, we have

$$
T_{w_{1}}\left(\Lambda\left(w_{1}\right)\right)=\Lambda\left(w_{1}\right) \quad \text { and } \quad T_{w_{2}}\left(\Lambda\left(w_{2}\right)\right)=\Lambda\left(w_{2}\right) .
$$

Taking differencing between the above two equalities, we have

$$
T_{w_{1}}\left(\Lambda\left(w_{1}\right)\right)-T_{w_{2}}\left(\Lambda\left(w_{2}\right)\right)=\Lambda\left(w_{1}\right)-\Lambda\left(w_{2}\right) .
$$

Adding and subtracting the term $T_{w_{1}}\left(\Lambda\left(w_{2}\right)\right)$ in the above equality, we have

$$
\Lambda\left(w_{1}\right)-\Lambda\left(w_{2}\right)=T_{w_{1}}\left(\Lambda\left(w_{1}\right)\right)-T_{w_{1}}\left(\Lambda\left(w_{2}\right)\right)+T_{w_{1}}\left(\Lambda\left(w_{2}\right)\right)-T_{w_{2}}\left(\Lambda\left(w_{2}\right)\right) .
$$


Consequently,

$$
\begin{aligned}
\left|\Lambda\left(w_{1}\right)-\Lambda\left(w_{2}\right)\right| \leq & \left|T_{w_{1}}\left(\Lambda\left(w_{1}\right)\right)-T_{w_{1}}\left(\Lambda\left(w_{2}\right)\right)\right| \\
& +\left|T_{w_{1}}\left(\Lambda\left(w_{2}\right)\right)-T_{w_{2}}\left(\Lambda\left(w_{2}\right)\right)\right| .
\end{aligned}
$$

According to Proposition 1, the first term on the right-hand side of the above expression is bounded above by $O\left(\sigma^{\alpha-\varepsilon}\right)\left|\Lambda\left(w_{1}\right)-\Lambda\left(w_{2}\right)\right|$.

LEMMA 5. For all $|\lambda|=O\left(\sigma^{\alpha-1}\right)$ and $w_{1}, w_{2} \in \mathcal{B}$, we have

$$
\left|T_{w_{1}}(\lambda)-T_{w_{2}}(\lambda)\right|=O\left(\sigma^{\alpha}\right)\left|w_{1}-w_{2}\right|_{k, \beta} .
$$

According to Lemma 5, the second term on the right-hand side of (40) is bounded above by $O\left(\sigma^{\alpha}\right)\left|w_{1}-w_{2}\right|_{k, \beta}$. Therefore, we have

$$
\left|\Lambda\left(w_{1}\right)-\Lambda\left(w_{2}\right)\right| \leq O\left(\sigma^{\alpha-\varepsilon}\right)\left|\Lambda\left(w_{1}\right)-\Lambda\left(w_{2}\right)\right|+O\left(\sigma^{\alpha}\right)\left|w_{1}-w_{2}\right|_{k, \beta} .
$$

Consequently, we have that for $w_{1}, w_{2} \in \mathcal{B}$,

$$
\left|\Lambda\left(w_{1}\right)-\Lambda\left(w_{2}\right)\right|=O\left(\sigma^{\alpha}\right)\left|w_{1}-w_{2}\right|_{k, \beta}
$$

According to (29),

$$
\left|\mathcal{G}^{\prime}\left[\sigma \mathbf{C} w_{2}\right]\right|_{k, \beta}=O(1)
$$

The above approximation and (41) give

$$
\left|\Lambda\left(w_{1}\right)-\Lambda\left(w_{2}\right)\right| \times\left|\mathcal{G}^{\prime}\left[\sigma \mathbf{C} w_{2}\right]\right|_{k, \beta}=O\left(\sigma^{\alpha}\right)\left|w_{1}-w_{2}\right|_{k, \beta}
$$

Combining the above display with (38) and (39), we complete our proof.

Proof of TheOREM 3. (i) is a direct application of Proposition 2, contraction mapping theorem and the KKT condition (16). We proceed to the proof of (ii). Because $\xi^{*}$ is the fixed point of $\Xi$ in $\mathcal{B}$, we have

$$
\begin{aligned}
\Xi\left[\xi^{*}\right] & =\Lambda\left(\xi^{*}\right) \mathcal{G}^{\prime}\left[\sigma \mathbf{C} \xi^{*}\right] \\
& =\kappa \mathcal{K}\left(\mathcal{G}^{\prime}[\mathbf{0}]\right)^{-1} \sigma^{\alpha-1}(1+o(1))\left(\mathcal{G}^{\prime}[\mathbf{0}]+O_{k, \beta}\left(\sigma \xi^{*}\right)\right) \\
& =\left(1+o_{k, \beta}(1)\right) \frac{\kappa \mathcal{G}^{\prime}[\mathbf{0}]}{\mathcal{K}\left(\mathcal{G}^{\prime}[\mathbf{0}]\right)} \sigma^{\alpha-1} .
\end{aligned}
$$

To obtain the second equality in the above display, we use approximation in Lemma 2 on page 2792 and (29). 


\subsection{Proof of Theorem 1.}

Proof of Theorem 1. Recall in (7) we define $\mathcal{G}(\xi)=\mathcal{H}(\mathbf{J}(\xi))=\mathcal{H}\left(u_{\xi}\right)$ in the context of elliptic PDE. In what follows, we apply Theorem 2 to the functional $\mathcal{G}$. It is sufficient to verify Assumptions A1-A3 given Assumptions H1-H4 with $k=1$ and $0<\beta<1$ and the Hölder coefficient being $\beta$.

We first present two useful lemmas. The following lemma guarantees the existence and uniqueness of the Hölder continuous solution to the elliptic PDE.

LEMMA 6. Suppose that $U$ is a bounded domain with a $C^{2, \beta}$ boundary $\partial U$ for $0<\beta<1$. Assume that there exist positive constants $\delta$ and $M$ such that $\min _{x \in \bar{U}} a(x)>\delta>0$, and $|a|_{1, \beta} \leq M$, and $f \in C^{\beta}(\bar{U})$. Then the elliptic PDE

$$
\begin{cases}-\nabla \cdot(a(x) \nabla u(x))=f(x) & \text { for } x \in U ; \\ u(x)=0 & \text { for } x \in \partial U,\end{cases}
$$

has a unique solution in $C^{2, \beta}(\bar{U})$. Denote this solution by $u_{a, f}$, then

$$
\left|u_{a, f}\right|_{2, \beta} \leq \kappa(\delta, M, d, U)|f|_{\beta},
$$

where $\kappa(\delta, M, d, U)$ is a positive constant, depending only on $\delta, M, d$ and the domain $U$.

We will also need the following lemma on the stability of the solution.

LEMMA 7. Suppose that $U$ is a bounded domain with a $C^{2, \beta}$ boundary $\partial U$ for $0<\beta<1$. Let $a_{1}, a_{2}, f_{1}$ and $f_{2}$ be functions over the domain $U$ such that

$$
\begin{aligned}
\min _{x \in \bar{U}} a_{1}(x) \geq \delta, \quad \min _{x \in \bar{U}} a_{2}(x) \geq \delta, \\
\left|a_{1}\right|_{1, \beta},\left|a_{2}\right|_{1, \beta} \leq M, \quad \text { and } \quad f_{1}, f_{2} \in C^{\beta}(\bar{U}) .
\end{aligned}
$$

Then

$$
\left|u_{a_{1}, f_{1}}-u_{a_{2}, f_{2}}\right|_{2, \beta} \leq \tilde{\kappa}(\delta, M, d, U)\left\{\left|f_{1}-f_{2}\right|_{\beta}+\left|a_{1}-a_{2}\right|_{1, \beta}\left|f_{1}\right|_{\beta}\right\},
$$

where the constant $\tilde{\kappa}(\delta, M, d, U)$ depends only on $\delta, M, d$ and the domain $U$.

The Fréchet derivative $\mathcal{G}^{\prime}[w]$ has the following expression:

$$
\mathcal{G}^{\prime}[w](x)=a_{w}(x) \nabla g_{w}(x) \cdot \nabla u_{w}(x),
$$

where $a_{w}(x)=a_{0} e^{-w(x)}, u_{w} \in C^{2, \beta}(\bar{U})$ is the unique solution to

$$
\begin{cases}-\nabla \cdot\left(a_{w}(x) \nabla u_{w}(x)\right)=f(x) & \text { for } x \in U ; \\ u_{w}(x)=0 & \text { for } x \in \partial U,\end{cases}
$$


and $g_{w}(x) \in C^{2, \beta}(\bar{U})$ is the unique solution to

$$
\begin{cases}-\nabla \cdot\left(a_{w}(x) \nabla g_{w}(x)\right)=\mathcal{H}^{\prime}\left[u_{w}\right](x) & \text { for } x \in U \\ g_{w}(x)=0 & \text { for } x \in \partial U\end{cases}
$$

For $w_{1}, w_{2} \in C^{1, \beta}(\bar{U})$, we are going to establish an upper bound for $\mid \mathcal{G}^{\prime}\left[w_{1}\right]-$ $\left.\mathcal{G}^{\prime}\left[w_{2}\right]\right|_{1, \beta}$. Note that

$$
\begin{aligned}
\mathcal{G}^{\prime}\left[w_{1}\right] & (x)-\mathcal{G}^{\prime}\left[w_{2}\right](x) \\
= & \left(a_{w_{1}}(x)-a_{w_{2}}(x)\right) \nabla g_{w_{1}}(x) \cdot \nabla u_{w_{1}}(x) \\
& +a_{w_{2}} \nabla g_{w_{2}}(x) \nabla\left(u_{w_{1}}-u_{w_{2}}(x)\right) \\
& +a_{w_{2}}(x) \nabla\left(g_{w_{1}}(x)-g_{w_{2}}(x)\right) \cdot \nabla u_{w_{1}}(x) .
\end{aligned}
$$

Thus,

$$
\begin{aligned}
\mid \mathcal{G}^{\prime}\left[w_{1}\right] & -\left.\mathcal{G}^{\prime}\left[w_{2}\right]\right|_{1, \beta} \\
\leq & \left|\left(a_{w_{1}}-a_{w_{2}}\right) \nabla g_{w_{1}} \cdot \nabla u_{w_{1}}\right|_{1, \beta} \\
\quad & \quad\left|a_{w_{2}} \nabla g_{w_{2}} \nabla\left(u_{w_{1}}-u_{w_{2}}\right)\right|_{1, \beta}+\left|a_{w_{2}} \nabla\left(g_{w_{1}}-g_{w_{2}}\right) \cdot \nabla u_{w_{1}}\right|_{1, \beta} .
\end{aligned}
$$

We will establish upper bounds for the three terms on the right-hand side in the above expression separately. First, note that $a_{w_{k}}=a_{0} e^{-w_{k}}, k=1,2$. Thus, there exists a constant $\varepsilon_{0}>0$ such that for all $\left|w_{1}\right|_{1, \beta},\left|w_{2}\right|_{1, \beta} \leq \varepsilon_{0}$,

$$
\left|a_{w_{1}}-a_{w_{2}}\right|_{1, \beta} \leq \kappa_{0}\left|w_{1}-w_{2}\right|_{1, \beta} .
$$

Therefore,

$$
\begin{aligned}
\left|\left(a_{w_{1}}-a_{w_{2}}\right) \nabla g_{w_{1}} \cdot \nabla u_{w_{1}}\right|_{1, \beta} & \leq\left|a_{w_{1}}-a_{w_{2}}\right|_{1, \beta}\left|\nabla g_{w_{1}}\right|_{1, \beta}\left|\nabla u_{w_{1}}\right|_{1, \beta} \\
& \leq \kappa_{0}\left|w_{1}-w_{2}\right|_{1, \beta}\left|g_{w_{1}}\right|_{2, \beta}\left|u_{w_{1}}\right|_{2, \beta} .
\end{aligned}
$$

Now we present upper bounds for $\left|g_{w_{1}}\right|_{2, \beta}$ and $\left|u_{w_{1}}\right|_{2, \beta}$. Let $\varepsilon_{0}$ be sufficiently small such that for all $|w|_{1, \beta} \leq \varepsilon_{0}, \min _{x \in \bar{U}} a_{w}(x) \geq \frac{1}{2} \min _{x \in \bar{U}} a_{0}(x)$ and $\left|a_{w}\right|_{1, \beta} \leq$ $2\left|a_{0}\right|_{1, \beta}$. According to Lemma 6 , we have that for all $|w|_{1, \beta} \leq \delta_{0}$,

$$
\left|u_{w}\right|_{2, \beta} \leq \kappa(\delta, M, d, U)|f|_{\beta},
$$

where $\delta=\frac{\min _{x \in \bar{U}} a_{0}(x)}{2}$ and $M=2\left|a_{0}\right|_{1, \beta}$. Furthermore, according to Assumption $\mathrm{H} 1$, we have that for $\left|u_{w}-u_{0}\right|_{2, \beta} \leq \delta_{H}$ :

$$
\left|\mathcal{H}^{\prime}\left[u_{w}\right]\right|_{\beta} \leq\left|\mathcal{H}^{\prime}\left[u_{0}\right]\right|_{\beta}+\kappa_{H}\left|u_{w}-u_{0}\right|_{2, \beta} \leq\left|\mathcal{H}^{\prime}\left[u_{0}\right]\right|_{\beta}+\kappa_{H} \delta_{H} .
$$

Set $f=\mathcal{H}^{\prime}\left[u_{w}\right]$ in Lemma 6 , and we have

$$
\left|g_{w}\right|_{2, \beta} \leq \kappa(\delta, M, d, U)\left|\mathcal{H}^{\prime}\left[u_{w}\right]\right|_{\beta} \leq \kappa(\delta, M, d, U)\left(\left|\mathcal{H}^{\prime}\left[u_{0}\right]\right|_{\beta}+\kappa_{H} \delta_{H}\right) .
$$


Combine this with (46) and (47), we have that for $\left|w_{1}\right|_{1, \beta},\left|w_{2}\right|_{1, \beta} \leq \varepsilon_{0}$

$$
\left|\left(a_{w_{1}}-a_{w_{2}}\right) \nabla g_{w_{1}} \cdot \nabla u_{w_{1}}\right|_{1, \beta} \leq \kappa_{0}\left|w_{1}-w_{2}\right|_{1, \beta},
$$

with a possibly different $\kappa_{0}$. We proceed to the second term on the right-hand side of (44):

$$
\begin{aligned}
\left|a_{w_{2}} \nabla g_{w_{2}} \cdot \nabla\left(u_{w_{1}}-u_{w_{2}}\right)\right|_{1, \beta} & \leq\left|a_{w_{2}}\right|_{1, \beta}\left|\nabla g_{w_{2}}\right|_{1, \beta}\left|\nabla\left(u_{w_{1}}-u_{w_{2}}\right)\right|_{1, \beta} \\
& \leq\left|a_{w_{2}}\right|_{1, \beta}\left|g_{w_{2}}\right|_{2, \beta}\left|u_{w_{1}}-u_{w_{2}}\right|_{2, \beta} .
\end{aligned}
$$

For $\left|w_{2}\right|_{1, \beta} \leq \varepsilon_{0}$, we have $\left|a_{w_{2}}\right|_{1, \beta} \leq 2\left|a_{0}\right|_{1, \beta}$. Moreover, $\left|g_{w_{2}}\right|_{2, \beta}$ is bounded above by a constant according to (49). Therefore,

$$
\left|a_{w_{2}} \nabla g_{w_{2}} \cdot \nabla\left(u_{w_{1}}-u_{w_{2}}\right)\right|_{1, \beta} \leq \kappa_{0}\left|u_{w_{1}}-u_{w_{2}}\right|_{2, \beta},
$$

for a possibly different $\kappa_{0}$. Taking $a_{1}=a_{w_{1}}, a_{2}=a_{w_{2}}$, and $f_{1}=f_{2}=f$ in Lemma 7, we have

$$
\left|u_{w_{1}}-u_{w_{2}}\right|_{2, \beta} \leq \tilde{\kappa}(\delta, M, d, U)\left|a_{1}-a_{2}\right|_{1, \beta}|f|_{\beta} \leq \kappa_{0}\left|w_{1}-w_{2}\right|_{1, \beta} .
$$

(52) and (53) give

$$
\left|a_{w_{2}} \nabla g_{w_{2}} \cdot \nabla\left(u_{w_{1}}-u_{w_{2}}\right)\right|_{1, \beta} \leq \kappa_{0}^{2}\left|w_{1}-w_{2}\right|_{1, \beta} .
$$

We proceed to the third term on the right-hand side of (44):

$$
\begin{aligned}
\left|a_{w_{2}} \nabla\left(g_{w_{1}}-g_{w_{2}}\right) \cdot \nabla u_{w_{1}}\right|_{1, \beta} & \leq\left|a_{w_{2}}\right|_{1, \beta}\left|\nabla\left(g_{w_{1}}-g_{w_{2}}\right)\right|_{1, \beta}\left|\nabla u_{w_{1}}\right|_{1, \beta} \\
& \leq\left|a_{w_{2}}\right|_{1, \beta}\left|g_{w_{1}}-g_{w_{2}}\right|_{2, \beta}\left|u_{w_{1}}\right|_{2, \beta} .
\end{aligned}
$$

According to the definition of $a_{w_{2}}$ and (53), we have that for $\left|w_{1}\right|_{1, \beta},\left|w_{2}\right|_{1, \beta} \leq \varepsilon_{0}$,

$$
\left|a_{w_{2}} \nabla\left(g_{w_{1}}-g_{w_{2}}\right) \cdot \nabla u_{w_{1}}\right|_{1, \beta} \leq \kappa_{0}\left|g_{w_{1}}-g_{w_{2}}\right|_{2, \beta} .
$$

Motivated by the definition of $g_{w_{1}}$ and $g_{w_{2}}$, we take $f_{1}=\mathcal{H}^{\prime}\left[w_{1}\right], f_{2}=\mathcal{H}^{\prime}\left[w_{2}\right]$, $a_{1}=a_{w_{1}}$ and $a_{2}=a_{w_{2}}$ in Lemma 7 , then

$$
\begin{aligned}
& \left|g_{w_{1}}-g_{w_{2}}\right|_{2, \beta} \\
& \quad \leq \tilde{\kappa}(\delta, M, d, U)\left\{\left|\mathcal{H}^{\prime}\left[w_{1}\right]-\mathcal{H}^{\prime}\left[w_{2}\right]\right|_{\beta}+\left.\left|a_{w_{1}}-a_{w_{2}}\right|\right|_{1, \beta}\left|\mathcal{H}^{\prime}\left[w_{1}\right]\right|_{\beta}\right\} .
\end{aligned}
$$

According to Assumption $\mathrm{H} 1$, for $\left|w_{1}\right|_{1, \beta},\left|w_{2}\right|_{1, \beta} \leq \delta_{H}$, we have

$$
\left|\mathcal{H}^{\prime}\left[w_{1}\right]-\mathcal{H}^{\prime}\left[w_{2}\right]\right|_{\beta} \leq \kappa_{H}\left|w_{1}-w_{2}\right|_{1, \beta}
$$

(58), (45), (48) and (57) give

$$
\left|g_{w_{1}}-g_{w_{2}}\right|_{2, \beta} \leq \kappa_{0}\left|w_{1}-w_{2}\right|_{1, \beta} .
$$

The above inequality and (56) give

$$
\left|a_{w_{2}} \nabla\left(g_{w_{1}}-g_{w_{2}}\right) \cdot \nabla u_{w_{1}}\right|_{1, \beta} \leq \kappa_{0}^{2}\left|w_{1}-w_{2}\right|_{1, \beta} .
$$


We combine (44), (50), (54) and (59), and arrive at

$$
\left|\mathcal{G}^{\prime}\left[w_{1}\right]-\mathcal{G}^{\prime}\left[w_{2}\right]\right|_{1, \beta} \leq \kappa_{0}\left|w_{1}-w_{2}\right|_{1, \beta},
$$

for $\varepsilon_{0}$ sufficiently small, $\left|w_{1}\right|_{1, \beta},\left|w_{2}\right|_{1, \beta} \leq \varepsilon_{0}$ and a possibly different $\kappa_{0}$. Thus, Assumption $\mathrm{A} 1$ is satisfied with $k=1$. According to the definition of $\mathcal{G}^{\prime}$, Assumption A2 is a direct application of Assumption H2. Assumption A3 is the same Assumption $\mathrm{H} 4$ for $k=1$. Now we have already checked all the Assumptions A1-A3.

\section{APPENDIX: PROOF OF SUPPORTING LEMMAS}

Proof of Lemma 1. Note that the event $\left\{\xi-\mathbf{C} \xi^{*} \notin \mathcal{B}\right\}=\left\{\left|\xi-\mathbf{C} \xi^{*}\right|_{k, \beta}>\right.$ $\left.\sigma^{\alpha-1-\varepsilon}\right\}$ implies the event $\left\{|\xi|>\sigma^{\alpha-1-\varepsilon}-\left|\xi^{*}\right|_{k, \beta}\right\}$. According to Theorem 3, $\left|\xi^{*}\right|_{k, \beta}=O\left(\sigma^{\alpha-1}\right)$. Thus,

$$
\left\{\xi-\mathbf{C} \xi^{*} \notin \mathcal{B}\right\} \subset\left\{|\xi|_{k, \beta}>\varepsilon_{0} \sigma^{\alpha-1-\varepsilon}\right\},
$$

for a positive constant $\varepsilon_{0}$ and $\sigma$ sufficiently small. Recall the definition

$$
|\xi|_{k, \beta}=\sum_{l=1}^{k} \sup _{|\gamma|=l} \sup _{x \in \bar{U}}\left|D^{\gamma} \xi(x)\right|+\sup _{|\gamma|=k}\left[D^{\gamma} \xi\right]_{\beta} \text {. }
$$

Consequently,

$$
\begin{aligned}
\left\{\xi-\mathbf{C} \xi^{*} \notin \mathcal{B}\right\} & \subset \bigcup_{l=1}^{k}\left\{\sup _{|\gamma|=l} \sup _{x \in \bar{U}}\left|D^{\gamma} \xi(x)\right|>\frac{\sigma^{\alpha-1-\varepsilon}}{k+1}\right\} \cup\left\{\sup _{|\gamma|=l}\left[D^{\gamma} \xi\right]_{\beta}>\frac{\sigma^{\alpha-1-\varepsilon}}{k+1}\right\} \\
& =\bigcup_{l=1}^{k} \bigcup_{|\gamma|=l}\left\{\sup _{x \in \bar{U}}\left|D^{\gamma} \xi(x)\right|>\frac{\sigma^{\alpha-1-\varepsilon}}{k+1}\right\} \bigcup_{|\gamma|=l}\left\{\left[D^{\gamma} \xi\right]_{\beta}>\frac{\sigma^{\alpha-1-\varepsilon}}{k+1}\right\} .
\end{aligned}
$$

The equality in the above display is due to the fact that $\left\{\sup _{l=1}^{m} X_{l} \geq \eta\right\}=$ $\bigcup_{l=1}^{m}\left\{X_{l} \geq \eta\right\}$ for any random variable $X_{l}, l=1, \ldots, m$ and constant $\eta$. According to the above display, we arrive at a upper bound of probability:

$$
\begin{aligned}
\mathbb{P}\left(\xi-\mathbf{C} \xi^{*} \notin \mathcal{B}\right) \leq & \sum_{l=1}^{k} \sum_{|\gamma|=l} \mathbb{P}\left(\sup _{x \in \bar{U}}\left|D^{\gamma} \xi(x)\right|>\frac{\sigma^{\alpha-1-\varepsilon}}{k+1}\right) \\
& +\sum_{|\gamma|=l} \mathbb{P}\left(\left[D^{\gamma} \xi\right]_{\beta}>\frac{\sigma^{\alpha-1-\varepsilon}}{k+1}\right) .
\end{aligned}
$$

We establish upper bounds for $\mathbb{P}\left(\sup _{x \in \bar{U}}\left|D^{\gamma} \xi(x)\right|>\frac{\sigma^{\alpha-1-\varepsilon}}{k+1}\right)$ and $\mathbb{P}\left(\left[D^{\gamma} \xi\right]_{\beta}>\right.$ $\left.\frac{\sigma^{\alpha-1-\varepsilon}}{k+1}\right)$ separately. We first analyze the term $\mathbb{P}\left(\sup _{x \in \bar{U}}\left|D^{\gamma} \xi(x)\right|>\frac{\sigma^{\alpha-1-\varepsilon}}{k+1}\right)$. We will need the following lemma, known as the Borell-TIS inequality, which was proved independently by Borell (1975) and Cirel'son, Ibragimov and Sudakov (1976). 
LEMMA 8 (Borell-TIS inequality). Let $g(x)$ be a centered and almost surely bounded Gaussian random field. Then $\mathbf{E} \sup _{x \in U}|g(x)|<\infty$. Furthermore, for any $t>\mathbf{E} \sup _{x \in U}|g(x)|$, we have

$$
\mathbb{P}\left(\sup _{x \in U}|g(x)|-\mathbf{E} \sup _{x \in U}|g(x)|>t\right) \leq 2 \exp \left\{-\frac{t^{2}}{2 \sup _{x \in U} \operatorname{Var}(g(x))}\right\} .
$$

According to Lemma 8, we have that for all $|\gamma| \leq k, \mathbf{E} \sup _{x \in \bar{U}}\left|D^{\gamma} \xi(x)\right|<\infty$ and

$$
\mathbb{P}\left(\sup _{x \in \bar{U}}\left|D^{\gamma} \xi(x)\right|>\frac{\sigma^{\alpha-1-\varepsilon}}{k+1}\right) \leq 2 \exp \left\{-\frac{\sigma^{2 \alpha-2-2 \varepsilon}}{8(k+1)^{2} \sup _{x \in \bar{U}} C_{D^{\gamma} \xi}(x, x)}\right\}
$$

for $\sigma$ sufficiently small such that $\sigma^{2 \alpha-2-2 \varepsilon}>2 \mathbf{E} \sup _{x \in \bar{U}}\left|D^{\gamma} \xi(x)\right|$, and $C_{D^{\gamma} \xi}$ is defined (14). According to Assumption A3, there exists a constant $\kappa_{0}$ such that for all $|\gamma| \leq k$,

$$
\sup _{x \in \bar{U}} C_{D^{\gamma} \xi}(x, x) \leq \sup _{y \in \bar{U}}\left|C_{D^{\gamma} \xi}(\cdot, y)\right|_{\beta}<\kappa_{0} .
$$

The above display together with (63) give

$$
\mathbb{P}\left(\sup _{x \in \bar{U}}\left|D^{\gamma} \xi(x)\right|>\frac{\sigma^{\alpha-1-\varepsilon}}{k+1}\right) \leq 2 \exp \left\{-\frac{\sigma^{2 \alpha-2-2 \varepsilon}}{8(k+1)^{2} \kappa_{0}}\right\}
$$

Combine this with (62), we have

(64) $\mathbb{P}\left(\xi-\mathbf{C} \xi^{*} \notin \mathcal{B}\right) \leq \kappa_{0} \exp \left\{-\frac{\sigma^{2 \alpha-2-2 \varepsilon}}{8(k+1)^{2} \kappa_{0}}\right\}+\sum_{|\gamma|=l} \mathbb{P}\left(\left[D^{\gamma} \xi\right]_{\beta}>\frac{\sigma^{\alpha-1-\varepsilon}}{k+1}\right)$,

for a possibly different $\kappa_{0}$ such that $\kappa_{0} \geq 2 \operatorname{Card}\{\gamma:|\gamma| \leq k\}$. We proceed to establishing upper bounds for $\mathbb{P}\left(\left[D^{\gamma} \xi\right]_{\beta}>\frac{\sigma^{\alpha-1-\varepsilon}}{k+1}\right),|\gamma|=k$. Recall that

$$
\left[D^{\gamma} \xi\right]_{\beta}=\sup _{x, y \in \bar{U}, x \neq y} \frac{\left|D^{\gamma} \xi(x)-D^{\gamma} \xi(y)\right|}{|x-y|^{\beta}} .
$$

Motivated by this definition, we define another centered Gaussian random field double indexed by $x, y \in \bar{U}$

$$
g(x, y)= \begin{cases}\frac{D^{\gamma} \xi(x)-D^{\gamma} \xi(y)}{|x-y|^{\beta}} & \text { for } x \neq y, \\ 0 & \text { for } x=y .\end{cases}
$$

According to Assumption A3 $\xi \in C^{k, \beta}(\bar{U})$ almost surely. Thus, $g(\cdot, \cdot)$ is bounded almost surely. According to Lemma 8, we have that $\operatorname{Esup}_{x, y \in \bar{U}, x \neq y}|g(x, y)|<\infty$, and

$$
\mathbb{P}\left(\sup _{x, y \in \bar{U}}|g(x, y)|>\frac{\sigma^{\alpha-1-\varepsilon}}{k+1}\right) \leq 2 \exp \left\{-\frac{\sigma^{2 \alpha-2-2 \varepsilon}}{8(k+1)^{2} \sup _{x, y \in \bar{U}} \operatorname{Var} g(x, y)}\right\},
$$


for $\sigma$ sufficiently small such that $\sigma^{2 \alpha-2-2 \varepsilon}>2 \mathbf{E} \sup _{x, y \in \bar{U}}|g(x, y)|$. The variance of $g(x, y)$ in the above expression is bounded above as follows:

$$
\begin{aligned}
\operatorname{Var} g(x, y) & =|x-y|^{-2 \beta}\left\{C_{D^{\gamma} \xi}(x, x)-C_{D^{\gamma} \xi}(x, y)+C_{D^{\gamma} \xi}(y, y)-C_{D^{\gamma} \xi}(x, y)\right\} \\
& \leq\left[C_{D^{\gamma} \xi}(x, \cdot)\right]_{2 \beta}+\left[C_{D^{\gamma} \xi}(y, \cdot)\right]_{2 \beta},
\end{aligned}
$$

which is bounded above by a constant $\kappa_{0}$ according to Assumption A3. Thus, we have

$$
\mathbb{P}\left(\sup _{x, y \in \bar{U}}|g(x, y)|>\frac{\sigma^{\alpha-1-\varepsilon}}{k+1}\right) \leq 2 \exp \left\{-\frac{\sigma^{2 \alpha-2-2 \varepsilon}}{8(k+1)^{2} \kappa_{0}}\right\} .
$$

Note that $\left[D^{\gamma} \xi\right]_{\beta}=\sup _{x, y \in \bar{U}}|g(x, y)|$. Therefore, the above display is equivalent to

$$
\mathbb{P}\left(\left[D^{\gamma} \xi\right]_{\beta}>\frac{\sigma^{\alpha-1-\varepsilon}}{k+1}\right) \leq 2 \exp \left\{-\frac{\sigma^{2 \alpha-2-2 \varepsilon}}{8(k+1)^{2} \kappa_{0}}\right\} .
$$

We conclude our proof by combining the above inequality with (64).

PROOF OF Lemma 2. Because $\Lambda(w)$ is a fixed point of $T_{w}(\cdot)$, this lemma is a direct application of (37).

Proof of Lemma 3. We define a function $h:[0,1] \rightarrow \mathbb{R}$,

$$
h(s)=\mathcal{G}\left(w_{2}+s\left(w_{1}-w_{2}\right)\right)-\mathcal{G}\left(w_{2}\right)-s \int_{U} \mathcal{G}^{\prime}\left[w_{2}\right](x)\left\{w_{1}(x)-w_{2}(x)\right\} d x .
$$

Notice that $h(0)=0$ and $h(1)=\mathcal{G}\left(w_{1}\right)-\mathcal{G}\left(w_{2}\right)-\int_{U} \mathcal{G}^{\prime}\left[w_{2}\right](x)\left(w_{1}(x)-\right.$ $\left.w_{2}(x)\right) d x$. Apply mean value theorem to $h$, and we have

(67) $\mathcal{G}\left(w_{1}\right)-\mathcal{G}\left(w_{2}\right)-\int_{U} \mathcal{G}^{\prime}\left[w_{2}\right](x)\left(w_{1}(x)-w_{2}(x)\right) d x=h(1)-h(0)=h^{\prime}(\tilde{s})$,

for some $\tilde{s} \in[0,1]$. According to the definition of Fréchet derivative, it is easy to check that

$$
h^{\prime}(s)=s \int_{U}\left\{\mathcal{G}^{\prime}\left[w_{1}+s\left(w_{1}-w_{2}\right)\right](x)-\mathcal{G}^{\prime}\left[w_{2}\right](x)\right\}\left(w_{1}(x)-w_{2}(x)\right) d x .
$$

Furthermore, we have

$$
\begin{aligned}
\mid s \int_{U} & \left\{\mathcal{G}^{\prime}\left[w_{1}+s\left(w_{1}-w_{2}\right)\right](x)-\mathcal{G}^{\prime}\left[w_{2}\right](x)\right\}\left(w_{1}(x)-w_{2}(x)\right) d x \mid \\
& \leq \operatorname{meas}(U)\left|w_{1}-w_{2}\right|_{0} \times\left|\mathcal{G}^{\prime}\left[w_{1}+s\left(w_{1}-w_{2}\right)\right](x)-\mathcal{G}^{\prime}\left[w_{2}\right](x)\right|_{0} \\
& \leq \operatorname{meas}(U)\left|w_{1}-w_{2}\right|_{0} \times \kappa_{G}\left|w_{1}-w_{2}\right|_{k, \beta} \\
& \leq \operatorname{meas}(U)\left|w_{1}-w_{2}\right|_{k, \beta}^{2} .
\end{aligned}
$$


Here, meas $(U)$ is the Lebesgue measure of the set $U$, the second inequality is due to Assumption A1 and the third inequality is due to the fact that $w,|w|_{0} \leq|w|_{k, \beta}$. Combine the above inequality and (67) we obtain the desired result.

PROOF OF LEMMA 4. We prove the lemma by induction. We first prove this lemma for the case where $k=0$ and $\beta>0$. We consider the conditional random field $\left\{\xi(x), x \in \bar{U} \mid Z_{1}=z\right\}$. It can be shown that there exists a continuous Gaussian random field, denoted by $\{\chi(x), x \in \bar{U}\}$, who has the same distribution as $\left\{\xi(x), x \in \bar{U} \mid Z_{1}=z\right\}$ and belongs to $C^{\beta}(\bar{U})$ almost surely. The mean and covariance function of $\chi(x)$ satisfy

$$
\begin{aligned}
\mu_{\chi}(x) & =\operatorname{Var}\left(Z_{1}\right)^{-1} \operatorname{Cov}\left(Z_{1}, \xi(x)\right) z=\operatorname{Var}\left(Z_{1}\right)^{-1} \int_{U} \xi^{*}(y) C(x, y) d y, \\
C_{\chi}(x, y) & =C(x, y)-\operatorname{Var}\left(Z_{1}\right)^{-1} \operatorname{Cov}\left(\xi(x), Z_{1}\right) \operatorname{Cov}\left(\xi(y), Z_{1}\right) \\
& =C(x, y)-\operatorname{Var}\left(Z_{1}\right)^{-1} \int_{U} \xi^{*}(z) C(x, z) d z \int_{U} \xi^{*}(z) C(y, z) d z .
\end{aligned}
$$

According to the expression (23) and $\sup _{y \in \bar{U}}|C(\cdot, y)|_{2 \beta} \in<\infty$, we have that

$$
\left|\mu_{\chi}\right|_{\beta}=O\left(\sigma^{1-\alpha} z\right) \text { and } \sup _{y \in \bar{U}}\left|C_{\chi}(, y)\right|_{2 \beta}<\infty .
$$

Let $\zeta(x)=\chi(x)-\mu_{\chi}(x)$ be a centered Gaussian random field. Then event $\left\{|\chi|_{\beta}^{2}>\right.$ $\left.\frac{\Lambda\left(\xi^{*}\right)^{-1} z}{\sigma}\right\}$ implies that $\left\{|\zeta|_{\beta}>\left(\frac{\Lambda\left(\xi^{*}\right)^{-1} z}{\sigma}\right)^{\frac{1}{2}}-\left|\mu_{\chi}\right|_{\beta}\right\}$. Furthermore, according to (68) and Lemma 2 on page 2792, we have

$$
\left\{|\chi|_{\beta}^{2}>\frac{\Lambda\left(\xi^{*}\right)^{-1} z}{\sigma}\right\} \subset\left\{|\zeta|_{\beta}>\varepsilon_{0} \sigma^{-\frac{\alpha}{2}} \sqrt{z}-O\left(\sigma^{1-\alpha} z\right)\right\} .
$$

Because $z \leq \kappa_{0} \sigma^{2 \alpha-2-\varepsilon}$, we have $\sigma^{-\frac{\alpha}{2}} \sqrt{z}-O\left(\sigma^{1-\alpha} z\right) \geq \varepsilon_{0} \sigma^{-\frac{\alpha}{2}} \sqrt{z}$ for a possibly different $\varepsilon_{0}$. Therefore,

$$
\left\{|\chi|_{\beta}^{2}>\frac{\Lambda\left(\xi^{*}\right)^{-1} z}{\sigma}\right\} \subset\left\{|\zeta|_{\beta}>\varepsilon_{0} \sigma^{-\frac{\alpha}{2}} \sqrt{z}\right\} .
$$

Consequently, we have

$$
\mathbb{P}\left(|\chi|_{\beta}^{2}>\frac{\Lambda\left(\xi^{*}\right)^{-1} z}{\sigma}\right) \leq \mathbb{P}\left(|\zeta|_{\beta}>\varepsilon_{0} \sigma^{-\frac{\alpha}{2}} \sqrt{z}\right) .
$$

According to the definition of the norm $|\zeta|_{\beta}=\sup _{x \in \bar{U}}|\zeta(x)|+[\zeta]_{\beta}$. Therefore, an upper bound for (69) is

$$
\begin{aligned}
\mathbb{P}\left(|\chi|_{\beta}^{2}>\frac{\Lambda\left(\xi^{*}\right)^{-1} z}{\sigma}\right) \leq & \mathbb{P}\left(\sup _{x \in \bar{U}}|\zeta(x)| \geq \frac{\varepsilon_{0}}{2} \sigma^{-\frac{\alpha}{2}} \sqrt{z}\right) \\
& +\mathbb{P}\left([\zeta]_{\beta} \geq \frac{\varepsilon_{2}}{2} \sigma^{-\frac{\alpha}{2}} \sqrt{z}\right) .
\end{aligned}
$$


We will present upper bounds for the first and second terms in the above display separately. We start with the first term. Because $\zeta$ is a centered and continuous Gaussian random field, with the aid of Lemma 8, we have that $\mathbf{E} \sup _{x \in \bar{U}}|\zeta(x)|<$ $\infty$ and

$$
\mathbb{P}\left(\sup _{x \in \bar{U}}|\zeta(x)|>\frac{\varepsilon_{0}}{2} \sigma^{-\frac{\alpha}{2}} \sqrt{z}\right) \leq 2 \exp \left\{-\frac{\varepsilon_{0}^{2} \sigma^{-\alpha} z}{32 \sup _{x \in \bar{U}} \operatorname{Cov}_{\chi}(x, x)}\right\},
$$

for $\sigma$ and $z$ such that $\varepsilon_{0} \sigma^{-\frac{\alpha}{2}} \sqrt{z}>2 \mathbf{E} \sup _{x \in \bar{U}}|\zeta(x)|$. Because $z \geq \sigma^{\varepsilon}, \varepsilon_{0} \sigma^{-\frac{\alpha}{2}} \sqrt{z}>$ $2 \mathbf{E} \sup _{x \in \bar{U}}|\zeta(x)|$ is satisfied for $\sigma$ sufficiently small. Consequently, for $\sigma$ sufficiently small, we have

$$
\mathbb{P}\left(\sup _{x \in \bar{U}}|\zeta(x)|>\frac{\varepsilon_{0}}{2} \sigma^{-\frac{\alpha}{2}} \sqrt{z}\right)<e^{-\varepsilon_{0} \sigma^{-\alpha} z}
$$

for a sufficiently small and possibly different $\varepsilon_{0}$. We proceed to the second term on the right-hand side of (70). Because $\zeta \in C^{\beta}(\bar{U})$ almost surely, we obtain an upper bound for $\mathbb{P}\left([\zeta]_{\beta}>\frac{\varepsilon_{0}}{2} \sigma^{-\frac{\alpha}{2}} \sqrt{z}\right)$ using similar arguments as those for (66) on page 2803,

$$
\mathbb{P}\left([\zeta]_{\beta}>\frac{\varepsilon_{0}}{2} \sigma^{-\frac{\alpha}{2}} \sqrt{z}\right)<2 e^{-\varepsilon_{0} \sigma^{-\alpha} z},
$$

for $\sigma$ sufficiently small and a positive constant $\varepsilon_{0}$. Combine (70), (71) and (72), we have

$$
\mathbb{P}\left(|\chi|_{\beta}^{2}>\frac{\Lambda\left(\xi^{*}\right)^{-1} z}{\sigma}\right)<2 e^{-\varepsilon_{0} \sigma^{-\alpha} z}
$$

Recall that $\chi$ has the same distribution as $\left\{\zeta(x): x \in \bar{U} \mid Z_{1}=z\right\}$, thus (73) implies

$$
\mathbb{P}\left(|\xi|_{\beta}^{2}>\frac{\Lambda\left(\xi^{*}\right)^{-1} z}{\sigma} \mid Z_{1}=z\right)<2 e^{-\varepsilon_{0} \sigma^{-\alpha} z}
$$

Using similar arguments, we have that for $\sigma$ sufficiently small

$$
\mathbb{P}\left(|\xi|_{\beta}^{2}>\frac{\Lambda\left(\xi^{*}\right)^{-1} z}{\sigma} \mid Z_{1}=-z\right)<2 e^{-\varepsilon_{0} \sigma^{-\alpha} z}
$$

Combing the above inequality with (74), we have

$$
\begin{gathered}
\mathbb{P}\left(|\xi|_{\beta}^{2}>\frac{\Lambda\left(\xi^{*}\right)^{-1} z}{\sigma} \mid Z_{1}=z\right)+\mathbb{P}\left(|\xi|_{\beta}^{2}>\frac{\Lambda\left(\xi^{*}\right)^{-1} z}{\sigma} \mid Z_{1}=-z\right) \\
<4 e^{-\varepsilon_{0} \sigma^{-\alpha} z}<e^{-\varepsilon_{0}^{\prime} \sigma^{-\alpha} z}
\end{gathered}
$$

for $\varepsilon_{0}^{\prime}<\varepsilon_{0}$ and $\sigma$ sufficiently small. This completes our proof for the case where $k=0$ and $\beta>0$. For the case $k=0$ and $\beta=0,|\xi|_{\beta}=|\xi|_{0}$. With similar proof as those for (71), we have

$$
\mathbb{P}\left(|\xi|_{0}^{2}>\frac{\Lambda\left(\xi^{*}\right)^{-1} z}{\sigma} \mid Z_{1}=z\right) \leq \mathbb{P}\left(\sup _{x \in \bar{U}}|\zeta(x)| \geq \frac{\varepsilon_{0}}{2} \sigma^{-\frac{\alpha}{2}} \sqrt{z}\right)<2 e^{-\varepsilon_{0} \sigma^{-\alpha} z}
$$


We also have similar results conditional on $Z_{1}=-z$. Therefore, for $\beta=0$ we also have

$$
\mathbb{P}\left(|\xi|_{\beta}^{2}>\frac{\Lambda\left(\xi^{*}\right)^{-1} z}{\sigma} \mid Z_{1}=z\right)+\mathbb{P}\left(|\xi|_{\beta}^{2}>\frac{\Lambda\left(\xi^{*}\right)^{-1} z}{\sigma} \mid Z_{1}=-z\right)<4 e^{-\varepsilon_{0} \sigma^{-\alpha} z}
$$

This completes our proof for the case that $k=0$. We now proceed to prove the lemma for $k \geq 1$. Assuming that for $k=m$,

$$
\begin{aligned}
& \mathbb{P}\left(|\xi|_{k, \beta}^{2}>\frac{\Lambda\left(\xi^{*}\right)^{-1} z}{\sigma} \mid Z_{1}=z\right)+\mathbb{P}\left(|\xi|_{k, \beta}^{2}>\frac{\Lambda\left(\xi^{*}\right)^{-1} z}{\sigma} \mid Z_{1}=-z\right) \\
& <e^{-\varepsilon_{0} \sigma^{-\alpha} z}
\end{aligned}
$$

for some positive constant $\varepsilon_{0}$ that is independent with $\sigma$ and $z$ but possibly depend on $k$. We will prove that the following inequality holds for $\sigma$ sufficiently small and a positive constant $\varepsilon_{0}$,

$$
\begin{aligned}
& \mathbb{P}\left(|\xi|_{m+1, \beta}^{2}>\frac{\Lambda\left(\xi^{*}\right)^{-1} z}{\sigma} \mid Z_{1}=z\right)+\mathbb{P}\left(|\xi|_{m+1, \beta}^{2}>\frac{\Lambda\left(\xi^{*}\right)^{-1} z}{\sigma} \mid Z_{1}=-z\right) \\
& \quad<e^{-\varepsilon_{0}^{\prime} \sigma^{-\alpha} z}
\end{aligned}
$$

According to the definition of the norm $|\cdot|_{m+1, \beta}$, we know that for $\beta>0$

$$
|\xi|_{m+1, \beta}=|\xi|_{m}+\sup _{|\gamma|=m+1} \sup _{x \in \bar{U}}\left|D^{\gamma} \xi(x)\right|+\sup _{|\gamma|=m+1}\left[D^{\gamma} \xi\right]_{\beta} .
$$

Therefore,

$$
\begin{aligned}
\left\{|\xi|_{m+1, \beta}^{2} \geq \frac{\Lambda\left(\xi^{*}\right)^{-1} z}{\sigma}\right\} & \\
\subset & \left\{|\xi|_{m}^{2} \geq \frac{\Lambda\left(\xi^{*}\right)^{-1} z}{2 \sigma}\right\} \\
& \cup\left(\bigcup_{|\gamma|=m+1} \bigcup_{\left|\gamma^{\prime}\right|=m+1}\left\{\left(\sup _{x \in \bar{U}}\left|D^{\gamma} \xi(x)\right|+\left[D^{\gamma^{\prime} \xi}\right]_{\beta}\right)^{2} \geq \frac{\Lambda\left(\xi^{*}\right)^{-1} z}{2 \sigma}\right\}\right) .
\end{aligned}
$$

Consequently, we arrive at an upper bound

$$
\begin{aligned}
\mathbb{P}\left(|\xi|_{m+1, \beta}^{2} \geq \frac{\Lambda\left(\xi^{*}\right)^{-1} z}{\sigma} \mid Z_{1}=z\right) \\
\leq \mathbb{P}\left(|\xi|_{m}^{2} \geq \frac{\Lambda\left(\xi^{*}\right)^{-1} z}{2 \sigma} \mid Z_{1}=z\right) \\
\quad+\sum_{|\gamma|=m+1} \sum_{\left|\gamma^{\prime}\right|=m+1} \mathbb{P}\left(\left(\sup _{x \in \bar{U}}\left|D^{\gamma} \xi(x)\right|+\left[D^{\gamma^{\prime} \xi}\right]_{\beta}\right)^{2}\right. \\
\left.\geq \frac{\Lambda\left(\xi^{*}\right)^{-1} z}{2 \sigma} \mid Z_{1}=z\right),
\end{aligned}
$$


We present upper bounds for the first and second terms on the right-hand side of the above display separately. For the first term, according to (77), we have

$$
\mathbb{P}\left(|\xi|_{m}^{2} \geq \frac{\Lambda\left(\xi^{*}\right)^{-1} z}{2 \sigma} \mid Z_{1}=z\right) \leq e^{-\varepsilon_{0} \sigma^{-\alpha} z}
$$

For the second term, notice that

$$
\begin{aligned}
& \left\{\left(\sup _{x \in \bar{U}}\left|D^{\gamma} \xi(x)\right|+\left[D^{\left.\gamma^{\prime} \xi\right]_{\beta}}\right)^{2} \geq \frac{\Lambda\left(\xi^{*}\right)^{-1} z}{2 \sigma}\right\}\right. \\
& \quad=\left\{\sup _{x \in \bar{U}}\left|D^{\gamma} \xi(x)\right|+\left[D^{\gamma^{\prime}} \xi\right]_{\beta} \geq \sqrt{\frac{\Lambda\left(\xi^{*}\right)^{-1} z}{2 \sigma}}\right\} \\
& \quad \subset\left\{\sup _{x \in \bar{U}}\left|D^{\gamma} \xi(x)\right| \geq \frac{1}{2} \sqrt{\frac{\Lambda\left(\xi^{*}\right)^{-1} z}{2 \sigma}}\right\} \cup\left\{\left[D^{\gamma^{\prime} \xi}\right]_{\beta} \geq \frac{1}{2} \sqrt{\frac{\Lambda\left(\xi^{*}\right)^{-1} z}{2 \sigma}}\right\} .
\end{aligned}
$$

Therefore,

$$
\begin{aligned}
& \mathbb{P}\left(\left(\sup _{x \in \bar{U}}\left|D^{\gamma} \xi(x)\right|+\left[D^{\gamma^{\prime}} \xi\right]_{\beta}\right)^{2} \geq \frac{\Lambda\left(\xi^{*}\right)^{-1} z}{2 \sigma} \mid Z_{1}=z\right) \\
& \leq \mathbb{P}\left(\sup _{x \in \bar{U}}\left|D^{\gamma} \xi(x)\right| \geq \frac{1}{2} \sqrt{\frac{\Lambda\left(\xi^{*}\right)^{-1} z}{2 \sigma}} \mid Z_{1}=z\right) \\
& +\mathbb{P}\left(\left[D^{\gamma^{\prime} \xi}\right]_{\beta} \geq \frac{1}{2} \sqrt{\frac{\Lambda\left(\xi^{*}\right)^{-1} z}{2 \sigma}} \mid Z_{1}=z\right) .
\end{aligned}
$$

Now we present upper bounds for the two terms on the right-hand side of the above inequality for $\gamma$ and $\gamma^{\prime}$ such that $|\gamma|=m+1$ and $\left|\gamma^{\prime}\right|=m+1$. To do so, we consider a continuous Gaussian random field $\chi_{1}$ that belongs to $C^{\beta}(\bar{U})$ almost surely, and it has the same distribution as $\left\{D^{\gamma} \xi(x), x \in \bar{U} \mid Z_{1}=z\right\}$.

LEMMA 9. Let $C_{\chi_{1}}(s, t)=\mathbf{E} \chi_{1}(s) \chi_{1}(t)$ and $\mu_{\chi_{1}}(t)=\mathbf{E} \chi_{1}(t)$, then we have

$$
\left|\mu_{\chi_{1}}\right|_{\beta}=O\left(\sigma^{1-\alpha} z\right) \quad \text { and } \sup _{y \in \bar{U}}\left|C_{\chi_{1}}(\cdot, y)\right|<\infty .
$$

The above expressions are uniform in $\gamma$ for $|\gamma|=m+1$.

Notice that the above lemma has the same form as (68), so with similar arguments as those for (71), we have

$$
\mathbb{P}\left(\sup _{x \in \bar{U}}\left|D^{\gamma} \chi_{1}(x)\right| \geq \frac{1}{2} \sqrt{\frac{\Lambda\left(\xi^{*}\right)^{-1} z}{2 \sigma}}\right) \leq e^{-\varepsilon_{0} \sigma^{-\alpha} z} .
$$


Also, similar as arguments before (72), we have

$$
\mathbb{P}\left(\left[D^{\gamma^{\prime}} \chi_{1}\right]_{\beta} \geq \frac{1}{2} \sqrt{\frac{\Lambda\left(\xi^{*}\right)^{-1} z}{2 \sigma}}\right) \leq e^{-\varepsilon_{0} \sigma^{-\alpha} z} .
$$

Combining (82) and (83) and (81), we have

$$
\mathbb{P}\left(\left(\sup _{x \in \bar{U}}\left|D^{\gamma} \xi(x)\right|+\left[D^{\gamma^{\prime}} \xi\right]\right)^{2} \geq \frac{\Lambda\left(\xi^{*}\right)^{-1} z}{2 \sigma} \mid Z_{1}=z\right) \leq 2 e^{-\varepsilon_{0} \sigma^{-\alpha} z} .
$$

Combining the above display with (79) and (80), we have

$$
\mathbb{P}\left(|\xi|_{m+1, \beta}^{2} \geq \frac{\Lambda\left(\xi^{*}\right)^{-1} z}{\sigma} \mid Z_{1}=z\right) \leq e^{-\varepsilon_{0} \sigma^{-\alpha} z}
$$

for $\sigma$ sufficiently small and a possibly different constant $\varepsilon_{0}$. Similarly, conditional on $Z_{1}=-z$, we have

$$
\mathbb{P}\left(|\xi|_{m+1, \beta}^{2} \geq \frac{\Lambda\left(\xi^{*}\right)^{-1} z}{\sigma} \mid Z_{1}=-z\right) \leq e^{-\varepsilon_{0} \sigma^{-\alpha} z}
$$

Thus,

$$
\begin{aligned}
& \mathbb{P}\left(|\xi|_{m+1, \beta}^{2} \geq \frac{\Lambda\left(\xi^{*}\right)^{-1} z}{\sigma} \mid Z_{1}=z\right)+\mathbb{P}\left(|\xi|_{m+1, \beta}^{2} \geq \frac{\Lambda\left(\xi^{*}\right)^{-1} z}{\sigma} \mid Z_{1}=-z\right) \\
& \quad \leq 2 e^{-\varepsilon_{0} \sigma^{-\alpha} z}
\end{aligned}
$$

and we complete the proof for (78) for the case where $\beta>0$. For $\beta=0,|\xi|_{m+1}=$ $|\xi|_{m}+\sup _{|\gamma|=m+1} \sup _{x \in \bar{U}}\left|D^{\gamma} \xi(x)\right|$. We obtain the proof for the case where $\beta=0$ by ignoring all the $\left[D^{\gamma^{\prime}} \xi\right]_{\beta}$ terms in the proof for the case where $\beta>0$. This completes the induction.

Proof of Lemma 6. According to Theorem 6.14 in Gilbarg and Trudinger (2015), we have that the PDE (42) has a unique solution in $C^{2, \beta}(\bar{U})$. Denote this solution by $u_{a, f}$, then according to Theorem 6.6 in Gilbarg and Trudinger (2015), we have the upper bound:

$$
\left|u_{a, f}\right|_{2, \beta} \leq \kappa(\delta, M, d, U)\left(\left|u_{a, f}\right|_{0}+|f|_{0}\right) .
$$

We conclude the proof with the following upper bound provided by Theorem 3.7 in Gilbarg and Trudinger (2015):

$$
\left|u_{a, f}\right|_{0} \leq \kappa_{0}|f|_{0}
$$

for a constant $\kappa_{0}$ depending only on the domain $U$ and $|a|_{1}$.

Proof OF Lemma 7. According to the definition of $u_{a_{1}, f_{1}}$ and $u_{a_{2}, f_{2}}$, we have that

$$
-\nabla \cdot\left(a_{1}(x) \nabla u_{a_{1}, f_{1}}(x)\right)=f_{1} \quad \text { and } \quad-\nabla \cdot\left(a_{2}(x) \nabla u_{a_{2}, f_{2}}(x)\right)=f_{2} .
$$


Taking difference between the above two equalities, we have

$$
-\nabla \cdot\left(a_{1} \nabla u_{a_{1}, f_{1}}\right)+\nabla \cdot\left(a_{2}(x) \nabla u_{a_{2}, f_{2}}\right)=f_{1}(x)-f_{2}(x) \quad \text { for } x \in U .
$$

Rearranging terms in the above expression, we have

$$
\begin{aligned}
-\nabla & \cdot\left(a_{2}(x) \nabla\left(u_{a_{2}, f_{2}}(x)-u_{a_{1}, f_{1}}(x)\right)\right) \\
& =f_{2}(x)-f_{1}(x)-\nabla \cdot\left\{\left(a_{1}(x)-a_{2}(x)\right) \nabla u_{a_{1}, f_{1}}(x)\right\} .
\end{aligned}
$$

Therefore, $\bar{u}=u_{a_{2}, f_{2}}-u_{a_{1}, f_{1}} \in C^{2, \beta}(\bar{U})$ is a solution to the elliptic PDE

$$
\begin{cases}-\nabla \cdot\left(a_{2}(x) \nabla \bar{u}(x)\right)=\bar{f}(x) & \text { for } x \in U ; \\ \bar{u}(x)=0 & \text { for } x \in \partial U,\end{cases}
$$

where $\bar{f}(x)=f_{2}(x)-f_{1}(x)-\nabla \cdot\left\{\left(a_{1}(x)-a_{2}(x)\right) \nabla u_{a_{1}, f_{1}}(x)\right\}$. According to Lemma 6, we have

$$
\left|u_{a_{2}, f_{2}}-u_{a_{1}, f_{1}}\right|_{2, \beta} \leq \kappa(\delta, M, d, U)|\bar{f}|_{\beta} .
$$

We further establish an upper bound for $|\bar{f}|_{\beta}$,

$$
|\bar{f}|_{\beta} \leq\left|f_{2}-f_{1}\right|_{\beta}+\left|a_{2}-a_{1}\right|_{1, \beta}\left|u_{a_{1}, f_{1}}\right|_{2, \beta} .
$$

According to Lemma 6,

$$
\left|u_{a_{1}, f_{1}}\right|_{2, \beta} \leq \kappa(\delta, M, d, U)\left|f_{1}\right|_{\beta} .
$$

Combining this with (84) and (85), we have

$\left|u_{a_{2}, f_{2}}-u_{a_{1}, f_{1}}\right|_{2, \beta} \leq \kappa(\delta, M, d, U)\left\{\left|f_{2}-f_{1}\right|_{\beta}+\kappa(\delta, M, d, U)\left|a_{2}-a_{1}\right|_{1, \beta}\left|f_{1}\right|_{\beta}\right\}$.

We complete the proof by setting $\tilde{\kappa}(\delta, M, d, U)=\max (\kappa(\delta, M, d, U)$, $\left.\kappa(\delta, M, d, U)^{2}\right)$.

ProOF OF LEMma 5. We take difference between $T_{w_{1}}(\lambda)$ and $T_{w_{2}}(\lambda)$,

$$
T_{w_{1}}(\lambda)-T_{w_{2}}(\lambda)=-\mathcal{K}\left(\mathcal{G}^{\prime}[\mathbf{0}]\right)^{-1} \sigma^{-1}\left\{\mathcal{G}\left(\sigma \mathbf{C} \lambda \mathcal{G}^{\prime}\left[\sigma \mathbf{C} w_{1}\right]\right)-\mathcal{G}\left(\sigma \mathbf{C} \lambda \mathcal{G}^{\prime}\left[\sigma \mathbf{C} w_{2}\right]\right)\right\}
$$

Therefore,

(86) $\left|T_{w_{1}}(\lambda)-T_{w_{2}}(\lambda)\right|=O\left(\sigma^{-1}\left\{\left|\mathcal{G}\left(\sigma \mathbf{C} \lambda \mathcal{G}^{\prime}\left[\sigma \mathbf{C} w_{1}\right]\right)-\mathcal{G}\left(\sigma \mathbf{C} \lambda \mathcal{G}^{\prime}\left[\sigma \mathbf{C} w_{2}\right]\right)\right|\right\}\right)$.

According to Lemma 3, we have

$$
\begin{aligned}
\mathcal{G}\left(\sigma \lambda \mathbf{C} \mathcal{G}^{\prime}\left[\sigma \mathbf{C} w_{1}\right]\right)-\mathcal{G}\left(\sigma \lambda \mathbf{C} \mathcal{G}^{\prime}\left[\sigma \mathbf{C} w_{2}\right]\right) \\
=\sigma \lambda \int_{U} \mathcal{G}^{\prime}\left[\sigma \lambda \mathbf{C} \mathcal{G}^{\prime}\left[\sigma \mathbf{C} w_{2}\right]\right](x) \mathbf{C}\left\{\mathcal{G}^{\prime}\left[\sigma \mathbf{C} w_{1}\right](x)-\mathcal{G}^{\prime}\left[\sigma \mathbf{C} w_{2}\right](x)\right\} d x \\
\quad+O\left(\sigma^{2} \lambda^{2}\left|\mathcal{G}^{\prime}\left[\sigma \mathbf{C} w_{1}\right]-\mathcal{G}^{\prime}\left[\sigma \mathbf{C} w_{2}\right]\right|_{k, \beta}^{2}\right) .
\end{aligned}
$$


According to (29) and Assumption A1, the above display can be further simplified as

$$
\mathcal{G}\left(\sigma \lambda \mathbf{C} \mathcal{G}^{\prime}\left[\sigma w_{1}\right]\right)-\mathcal{G}\left(\sigma \lambda \mathbf{C G}^{\prime}\left[\sigma w_{2}\right]\right)=O\left(\sigma \lambda\left|\mathcal{G}^{\prime}\left[\sigma w_{1}\right]-\mathcal{G}^{\prime}\left[\sigma w_{2}\right]\right|_{k, \beta}\right),
$$

which is further simplified as

$$
\mathcal{G}\left(\sigma \lambda \mathbf{C G}^{\prime}\left[\sigma w_{1}\right]\right)-\mathcal{G}\left(\sigma \lambda \mathbf{C G}^{\prime}\left[\sigma w_{2}\right]\right)=O\left(\sigma \lambda \sigma\left|w_{1}-w_{2}\right|_{k, \beta}\right) .
$$

The above expression and (86) give

$$
\left|T_{w_{1}}(\lambda)-T_{w_{2}}(\lambda)\right|=O\left(\sigma \lambda\left|w_{1}-w_{2}\right|_{k, \beta}\right)=O\left(\sigma^{\alpha}\right)\left|w_{1}-w_{2}\right|_{k, \beta} .
$$

The last inequality in the above expression is due to $\lambda=O\left(\sigma^{\alpha-1}\right)$.

Proof of Lemma 9. We need the next lemma for the current proof.

LEMMA 10. We define the covariance function:

$$
C_{D^{\gamma} \xi, \xi}(x, y)=\operatorname{Cov}\left(D^{\gamma} \xi(x), \xi(y)\right) .
$$

Then $\sup _{y \in \bar{U}}\left|C_{D^{\gamma \xi}, \xi}(\cdot, y)\right|_{2 \beta}<\infty$ for all $|\gamma| \leq k$ under Assumption A3.

Now we compute the mean and covariance of $\chi_{1}$ :

$$
\begin{aligned}
\mu_{\chi_{1}}(x) & =\mathbb{E}\left[D^{\gamma} \xi(x) \mid Z_{1}=z\right] \\
& =\operatorname{Var}\left(Z_{1}\right)^{-1} \operatorname{Cov}\left(D^{\gamma} \xi(x), Z_{1}\right) z \\
& =\operatorname{Var}\left(Z_{1}\right)^{-1} \int_{U} C_{D^{\gamma} \xi, \xi}(x, y) \xi^{*}(y) d y z,
\end{aligned}
$$

and

$$
\begin{aligned}
C_{\chi_{1}}(x, y) & =C_{D^{\gamma} \xi}(x, y)-\operatorname{Var}\left(Z_{1}\right)^{-1} \operatorname{Cov}\left(D^{\gamma} \xi(x), Z_{1}\right) \operatorname{Cov}\left(D^{\gamma} \xi(y), Z_{1}\right) \\
& =C_{D^{\gamma} \xi}(x, y)-\frac{\int_{U} C_{D^{\gamma} \xi, \xi}(x, r) \xi^{*}(y) d r \int_{U} C_{D^{\gamma} \xi, \xi}(y, r) \xi^{*}(y) d r}{\operatorname{Var}\left(Z_{1}\right)} .
\end{aligned}
$$

Recall that $\operatorname{Var}\left(Z_{1}\right) \geq \varepsilon_{0} \sigma^{2 \alpha-2}$ for some positive constant $\varepsilon_{0}$, and $\left|\xi^{*}\right|_{k, \beta}=$ $O\left(\sigma^{\alpha-1}\right)$. With the aid of Lemma 10 , we simplify the mean and covariance of $\chi_{1}$ :

$$
\left|\mu_{\chi_{1}}\right|_{\beta}=O\left(\sigma^{2-2 \alpha}\left|\xi^{*}\right|_{0} \sup _{y}\left|C_{D^{\gamma} \xi, \xi}(\cdot, y)\right|_{2 \beta} z\right)=O\left(\sigma^{1-\alpha} z\right),
$$

and

$$
\begin{aligned}
\sup _{y \in \bar{U}}\left|C_{\chi_{1}}(\cdot, y)\right|_{2 \beta} & =O\left(\sup _{y \in \bar{U}}\left|C_{D^{\gamma} \xi}(\cdot, y)\right|_{2 \beta}+\sigma^{2-2 \alpha}\left|\xi^{*}\right|_{0}^{2} \sup _{y \in \bar{U}}\left|C_{D^{\gamma \xi}, \xi}(\cdot, y)\right|_{2 \beta}^{2}\right) \\
& =O(1) .
\end{aligned}
$$


Proof of Lemma 10. We will use induction to prove that for all $l=$ $0,1, \ldots, k,|\gamma|=l$,

$$
\sup _{y \in \bar{U}}\left|C_{D^{\gamma} \xi, \xi}(\cdot, y)\right|_{2 \beta}<\infty .
$$

To start with, for $l=0$ and $|\gamma|=l$, (87) holds because of Assumption A3 and

$$
C_{D^{\gamma \xi}, \xi}(s, t)=C(s, t) \text {. }
$$

Suppose that for all $\left|\gamma^{\prime}\right|=l$,

$$
\sup _{y \in \bar{U}}\left|C_{D^{\gamma^{\prime}} \xi, \xi}(\cdot, y)\right|_{k-l, 2 \beta}<\infty .
$$

For $|\gamma|=l+1$, we want to show that

$$
\sup _{y \in \bar{U}}\left|C_{D^{\gamma} \xi, \xi}(\cdot, y)\right|_{k-l-1,2 \beta}<\infty .
$$

Without loss of generality, we assume that $\gamma=\left(\gamma_{1}, \ldots, \gamma_{d}\right)$ and $\gamma_{1} \geq 1$. Let $e_{1}=$ $(1, \ldots, 0)$ be a $d$-dimensional basis vector, and $\gamma^{\prime}=\gamma-e_{1}$, then $\left|\gamma^{\prime}\right|=l$. We compute $C_{D^{\gamma \xi}, \xi}$ :

$$
\begin{aligned}
C_{D^{\gamma \xi}, \xi}(x, y) & =\lim _{\varepsilon_{1} \rightarrow 0} \operatorname{Cov}\left(\frac{D^{\gamma^{\prime}} \xi\left(x+\varepsilon_{1} e_{1}\right)-D^{\gamma^{\prime}} \xi(x)}{\varepsilon_{1}}, \xi(y)\right) \\
& =\lim _{\varepsilon_{1} \rightarrow 0} \varepsilon_{1}^{-1}\left\{C_{D^{\gamma^{\prime} \xi, \xi}}\left(x+\varepsilon_{1} e_{1}, y\right)-C_{D^{\gamma^{\prime} \xi, \xi}}(x, y)\right\} \\
& =\frac{\partial}{\partial x_{1}} C_{D^{\gamma^{\prime} \xi}}(x, y) .
\end{aligned}
$$

Consequently,

$$
\left|C_{D^{\gamma \xi}, \xi}(\cdot, y)\right|_{k-l-1,2 \beta}=\left|\frac{\partial}{\partial x_{1}} C_{D^{\gamma^{\prime} \xi}}(\cdot, y)\right|_{k-l-1,2 \beta} \leq\left|C_{D^{\gamma^{\prime} \xi}}(\cdot, y)\right|_{k-l, 2 \beta} .
$$

Thus,

$$
\sup _{y \in \bar{U}}\left|C_{D^{\gamma} \xi, \xi}(\cdot, y)\right|_{k-l-1,2 \beta} \leq \sup _{y \in \bar{U}}\left|C_{D^{\gamma^{\prime} \xi}}(\cdot, y)\right|_{k-l, 2 \beta}<\infty .
$$

The second inequality of the above display is due to (88). The lemma is proved by induction.

\section{REFERENCES}

AdLER, R. J. (1981). The Geometry of Random Fields. Wiley, Chichester. MR0611857 ADLER, R. J., BLANCHET, J. H. and LiU, J. C. (2008). Efficient simulation for tail probabilities of Gaussian random fields. In Proceeding of Winter Simulation Conference.

Adler, R. J., Blanchet, J. H. and LiU, J. (2012). Efficient Monte Carlo for high excursions of

Gaussian random fields. Ann. Appl. Probab. 22 1167-1214. MR2977989 
Adler, R. J. and TAYlor, J. E. (2007). Random Fields and Geometry. Springer, New York. MR2319516

ARMSTrong, S., KuUsi, T. and Mourrat, J.-C. (2017). Quantitative stochastic homogenization and large-scale regularity. ArXiv Preprint ArXiv:1705.05300.

AZAÏ S, J.-M. and WSChEBOR, M. (2008). A general expression for the distribution of the maximum of a Gaussian field and the approximation of the tail. Stochastic Process. Appl. 118 11901218. MR2428714

AZAïs, J. M. and Wschebor, M. (2009). Level Sets and Extrema of Random Processes and Fields. Wiley, Hoboken, NJ.

Berglund, N., Di Gesu, G. and Weber, H. (2017). An Eyring-Kramers law for the stochastic Allen-Cahn equation in dimension two. Electron. J. Probab. 22.

BERMAN, S. M. (1985). An asymptotic formula for the distribution of the maximum of a Gaussian process with stationary increments. J. Appl. Probab. 22 454-460.

Borell, C. (1975). The Brunn-Minkowski inequality in Gauss space. Invent. Math. 30 207-216. MR0399402

Borell, C. (2003). The Ehrhard inequality. C. R. Math. Acad. Sci. Paris 337 663-666. MR2030108

Charbeneau, R. J. (2000). Groundwater Hydraulics and Pollutant Transport. Prentice Hall.

Cirel'son, B. S., Ibragimov, I. A. and Sudakov, V. N. (1976). Norms of Gaussian sample functions. In Proceedings of the Third Japan-USSR Symposium on Probability Theory (G. Maruyama and J. V. Prokhorov, eds.) 20-41. Springer, Berlin.

Cirel'son, B. S., Ibragimov, I. A. and Sudakov, V. N. (1976). Norms of Gaussian sample functions. In Proceedings of the Third Japan-USSR Symposium on Probability Theory (Tashkent, 1975) Lecture Notes in Math. 550 20-41. Springer, Berlin. MR0458556

FREEZE, R. A. (1975). A stochastic-conceptual analysis of one-dimensional groundwater flow in nonuniform homogeneous media. Water Resour. Res. 11.

Gilbarg, D. and Trudinger, N. S. (2015). Elliptic Partial Differential Equations of Second Order. Springer.

LANDAU, H. J. and SHEPP, L. A. (1970). On the supremum of a Gaussian process. Sankhya A 32 369-378. MR0286167

Ledoux, M. and Talagrand, M. (1991). Probability in Banach Spaces: Isoperimetry and Processes. Ergebnisse der Mathematik und Ihrer Grenzgebiete 3. Folge 23. Springer, Berlin.

LI, X., LIU, J. and XU, G. (2016). On the tail probabilities of aggregated lognormal random fields with small noise. Math. Oper. Res. 41 236-246. MR3465750

LIU, J. (2012). Tail approximations of integrals of Gaussian random fields. Ann. Probab. 401069 1104. MR2962087

LIU, J., LU, J. and ZHOU, X. (2015). Efficient rare event simulation for failure problems in random media. SIAM J. Sci. Comput. 37 A609-A624.

LIU, J. and XU, G. (2012). Some asymptotic results of Gaussian random fields with varying mean functions and the associated processes. Ann. Statist. 40 262-293.

LIU, J. and ZHOU, X. (2013). On the failure probability of one dimensional random material under delta external force. Commun. Math. Sci. 11 499-521. MR3002562

LiU, J. and ZHOU, X. (2014). Extreme analysis of a random ordinary differential equation. J. Appl. Probab. 51 1021-1036.

MARCus, M. B. and ShePp, L. A. (1970). Continuity of Gaussian processes. Trans. Amer. Math. Soc. $151377-391$.

Piterbarg, V. I. (1996). Asymptotic Methods in the Theory of Gaussian Processes and Fields. Amer. Math. Soc., Providence, RI.

SUdAKOV, V. N. and TSIRELSON, B. S. (1974). Extremal properties of half spaces for spherically invariant measures. Zap. Nauchn. Sem. LOMI 45 75-82.

Sun, J. Y. (1993). Tail probabilities of the maxima of Gaussian random-fields. Ann. Probab. 21 $34-71$. 
Talagrand, M. (1996). Majorizing measures: The generic chaining. Ann. Probab. 24 1049-1103. MR1411488

TAYlor, J. E. and AdLER, R. J. (2003). Euler characteristics for Gaussian fields on manifolds. Ann. Probab. 31 533-563. MR1964940

TAYlor, J., TAKEMURA, A. and ADler, R. J. (2005). Validity of the expected Euler characteristic heuristic. Ann. Probab. 33 1362-1396.

XU, G., LIN, G. and LIU, J. (2014). Rare-event simulation for the stochastic Korteweg-de Vries equation. SIAM/ASA J. Uncertain. Quantificat. 2 698-716.

\section{LI}

School of Statistics

UNIVERSITY OF MINNESOTA

MinNEAPOLIS, MinNESOTA 55455

USA

E-MAIL: lixx1766@umn.edu

J. LU

DEPARTMENT OF PHYSICS

AND DEPARTMENT OF CHEMISTRY

DUKE UNIVERSITY

DURHAM, NORTH CAROLINA 27708

USA

E-MAIL: jianfeng@math.duke.edu
J. LIU

DEPARTMENT OF Statistics

COLUMBIA UNIVERSITY

NEW YORK, NEW YORK 10027

USA

E-MAIL: jcliu@stat.columbia.edu

X. ZHOU

DePARTMENT OF MATHEMATICS

City University OF HoNG Kong

TAT CHEE Ave, Kowloon

Hong Kong SAR

E-MAIL: xizhou@ cityu.edu.hk 